\title{
Desinflação Ótima na Presença de Inércia Inflacionária, Formação de Hábito e Fricções Monetárias*
}

\author{
Marco A. F. H. Cavalcanti ${ }^{\dagger}$
}

\author{
Conteúdo: 1. Introdução; 2. 0 Modelo; 3. Desinflação sob Regras 'Arbitrárias' de Política \\ Monetária; 4. Desinflação Ótima; 5. Conclusão; A. Derivação do Bloco de \\ Demanda; B. Derivação da Equação de Oferta; C. Derivação do Critério de \\ Bem-Estar; D. Derivação da Política Ótima de Desinflação. \\ Palavras-chave: Política Monetária; Desinflação; Regras Ótimas. \\ Códigos JEL: $\quad$ E31; E43; E52; E58.
}

Este artigo analisa, no contexto de um modelo monetário de equilíbrio geral nos moldes 'novo-keynesianos', as propriedades das políticas ótimas de desinflação, buscando determinar as condições sob as quais: (i) a trajetória ótima de desinflação envolve perdas substanciais de produto; (ii) uma estratégia de desinflação 'rápida' é preferível a uma desinflação 'gradual'. De acordo com os resultados obtidos, a existência de diferentes graus de fricções monetárias e de inércia no produto e na inflação permite justificar diferentes trajetórias ótimas de desinflação, algumas envolvendo queda rápida e indolor da inflação, outras associadas à lenta redução das taxas inflacionárias acompanhada de forte recessão.

This paper investigates the properties of optimal disinflation policies in a 'New-Keynesian' monetary general equilibrium model, seeking to identify conditions under which: (i) the optimal disinflationary path involves significant output losses; (ii) a 'rapid' disinflation is preferable to a 'gradual' one. According to our results, different degrees of monetary frictions and inertia in output or inflation may lead to different optimal disinflationary policies - some of which will be quick and painless, while others will proceed slowly and generate deep recessions.

\footnotetext{
* Baseado no Capítulo 2 da Tese de Doutorado defendida pelo autor na PUC-Rio. O autor agradece a valiosa orientação de Eduardo Loyo e Ilan Goldfajn.

† Pesquisador do IPEA e Professor da PUC-Rio. Endereço: IPEA - Av. Pres. Antonio Carlos, 51/1407, 20020-010, Rio de Janeiro - RJ. E-mail: E-mail: marco.cavalcanti@ipea.gov.br
} 


\section{INTRODUÇÃO}

Suponha que a taxa de inflação de determinada economia se encontre acima do nível ótimo de longo prazo, e que as autoridades monetárias desejem conduzir a economia para um novo equilíbrio com inflação mais baixa. Nesse novo equilíbrio, o nível de bem-estar da sociedade será, por definição, mais alto do que no equilíbrio original. Entretanto, é provável que a transição para o novo equilíbrio envolva custos de bem-estar não desprezíveis - associados, por exemplo, a perdas de produto ou variações excessivas na taxa de juros. A condução ótima da política monetária passa necessariamente, portanto, pela identificação da trajetória de desinflação que minimize, para a economia em questão, os custos de bem-estar na transição para o novo equilíbrio. O objetivo deste artigo é analisar, no contexto de um modelo monetário de equilíbrio geral nos moldes 'novo-keynesianos', as características das políticas ótimas de desinflação. Busca-se, em particular, contribuir para o melhor entendimento das condições sob as quais:

(i) a trajetória ótima de desinflação envolve perdas substanciais de produto;

(ii) uma estratégia de desinflação 'rápida' é preferível a uma desinflação 'gradual'.

O foco da análise será em processos de desinflação de 'pequena magnitude', tais como as desinflações observadas nos EUA entre 1979 e 1984 (quando a inflação passou de 13,3\% para 4\% ao ano) e no Brasil entre 2002 e 2006 (quando a inflação caiu de 12,5\% para 3,1\% ao ano). Em relação à extensa literatura teórica e empírica sobre 'pequenas desinflações', ${ }^{1}$ este artigo procura contribuir de três formas. Primeiro, a análise de bem-estar está calcada nos mesmos microfundamentos subjacentes às equações estruturais do modelo, e não em critérios relativamente arbitrários usados na maioria dos outros estudos - como, por exemplo, a 'taxa de sacrifício'. Segundo, analisam-se aspectos relativamente inexplorados na literatura, como o papel das fricções monetárias, e da interação destas com o grau de indexação da economia, na determinação da velocidade ótima de desinflação. Esta é uma questão potencialmente relevante, pois a análise de Woodford (2003, cap. 6) sugere que, na presença de friç̧ões monetárias, as variações da taxa de juros também acarretam efeitos sobre o bem estar; isso implica um dilema adicional a ser resolvido pelas autoridades monetárias, entre estabilizar a inflação ou a taxa de juros, que deve ser levado em consideração ao optar-se entre uma desinflação rápida ou gradual. Terceiro, a questão da desinflação é analisada a partir de regras baseadas no controle da taxa de juros pelo Banco Central, e não simplesmente na determinação de trajetórias para a evolução do estoque de moeda na economia, como feito na maioria dos trabalhos sobre o tema. Tendo em vista que, de acordo com a prática corrente na condução da política monetária, o principal instrumento de política é a taxa de juros básica, ${ }^{2}$ essa opção metodológica revela-se importante na aplicação dos resultados para fins de recomendações de política.

Deve-se ressaltar que a presente análise restringe-se a uma economia fechada e trata apenas de políticas de desinflação anunciadas inesperadamente e que sejam vistas como críveis e permanentes. Logo, desconsideram-se questões como o papel da taxa de câmbio e a evolução das expectativas dos agentes privados com relação à condução da política monetária (em um contexto de informação imperfeita e aprendizado), que podem proporcionar explicações pertinentes para certos fatos estilizados associados aos processos de desinflação, bem como análises interessantes das estratégias ótimas de desinflação. ${ }^{3}$

0 artigo está organizado em quatro seções, além desta introdução. A Seção 2 apresenta o modelo. As Seções 3 e 4 discutem as características das trajetórias de desinflação sob políticas monetárias 'ar-

\footnotetext{
${ }^{1}$ Ver, dentre outros, Ball (1994a,b), Ireland (1995, 1997), Danziger (1988), Calvo et alii (2003) e Erceg e Levin (2003).

${ }^{2}$ Ver a discussão a esse respeito em Woodford (2003, cap. 1).

${ }^{3}$ Calvo et alii (2003), dentre outros, analisam o papel da taxa de câmbio em processos de desinflação. Para algumas análises que abordam a questão da desinflação na presença de informação imperfeita, ver Ball (1995), Huh e Lansing (2000) e Erceg e Levin (2003).
} 
bitrárias' e políticas 'ótimas', respectivamente. Por fim, a Seção 5 resume os principais resultados da análise e tece as considerações finais.

\section{O MODELO}

\subsection{Visão Geral}

A estrutura do modelo é importada diretamente da literatura 'novo-keynesiana' sistematizada em Woodford (2003), que assenta no seguinte modelo 'básico' (log-linearizado em torno do equilíbrio em estado estacionário com inflação zero):

$$
\begin{aligned}
& y_{t}=E_{t} y_{t+1}-\sigma\left(r_{t}-E_{t} \pi_{t+1}\right)+\left(\xi_{t}-E_{t} \xi_{t+1}\right) \\
& \pi_{t}=\kappa\left(y_{t}-y_{t}^{n}\right)+\beta E_{t} \pi_{t+1}
\end{aligned}
$$

onde $y$ é o nível de produto na economia, $y^{n}$ o nível de produto 'natural' (isto é, o produto que seria observado no equilíbrio com flexibilidade perfeita de preços), $\pi$ a taxa de inflação, $r$ a taxa de juros nominal e $\xi$ um agregado de 'choques de demanda'. Todos os parâmetros são positivos, sendo $\sigma$ a elasticidade de substituição intertemporal no dispêndio privado, $\beta$ o fator de desconto intertemporal e $\kappa$ um parâmetro que depende da freqüência dos reajustes de preços na economia e da elasticidade do custo marginal real em relação ao produto.

A equação (1), que relaciona o produto da economia em cada momento aos níveis correntes e esperados para o futuro da taxa de juros e dos choques, é a equação de demanda agregada. Essa equação é usualmente denominada 'curva IS intertemporal' por analogia com a curva IS tradicional. A equação (2), que explica a inflação corrente pelos níveis esperados do hiato do produto corrente e futuro, é a curva de oferta agregada. Essa equação é conhecida como 'Curva de Phillips Novo-Keynesiana'. A derivação dessas equações baseia-se, respectivamente, em problemas de otimização intertemporal por parte de um consumidor e um produtor representativo.

Devido à sua simplicidade, esse modelo não é capaz de reproduzir diversas características comumente observadas em economias reais, tais como as respostas defasadas e graduais da inflação e do produto a choques monetários. ${ }^{4}$ Essa limitação do modelo novo-keynesiano básico inspirou várias extensões com o objetivo de tornar a análise mais 'realista', adequando-a aos fatos estilizados relativos aos efeitos da política monetária. No modelo apresentado a seguir, adotam-se duas dessas extensões: a presença de formação (ou persistência) de hábito no consumo, que introduz certo grau de persistência endógena no produto, e a indexação de preços não-otimizados à inflação passada, que gera inércia no processo inflacionário. ${ }^{5}$

O presente artigo também generaliza o modelo novo-keynesiano básico pela consideração explícita do papel da moeda na presença de friç̧ões transacionais, conforme feito, por exemplo, em Ireland (2001) e Woodford (2003, cap. 4). A relevância dessa modificação do modelo básico reside no fato de que, conforme mostra Woodford (2003, cap. 6), a presença de friç̧ões monetárias gera um motivo para a estabilização da taxa de juros - pois desvios da taxa de juros em relação a um nível crítico afetam negativamente o bem estar -; logo, introduz-se, na discussão das trajetórias ótimas de desinflação, um dilema adicional a ser resolvido pelas autoridades monetárias: estabilizar a inflação ou a taxa de juros. ${ }^{6}$

\footnotetext{
${ }^{4}$ Para uma resenha da literatura empírica relativa aos efeitos da política monetária, ver Christiano and Harrison (1999).

${ }^{5}$ Para aplicações da hipótese de formação de hábito à análise de política monetária ver Fuhrer (2000), Amato e Laubach (2003) e Boivin e Giannoni (2003). A hipótese de indexação de preços não-otimizados à inflação passada é adotada, por exemplo, em Christiano et alii (2001), Boivin e Giannoni (2003) e Woodford (2003).

${ }^{6}$ Rotemberg e Woodford (1997) e Woodford (2003, cap. 6) consideram uma razão adicional para a existência do dilema entre estabilização da inflação e da taxa de juros: tendo em vista que a taxa de juros nominal não pode ser negativa, a política
} 
Vale ressaltar, por fim, que o trabalho visa analisar apenas trajetórias determinísticas de desinflação. Por essa razão, desconsidera-se a ocorrência de distúrbios estocásticos de qualquer natureza, de modo a simplificar a análise. Em conseqüência dessa opção e da hipótese (discutida mais à frente) de eficiência do nível de produto em equilíbrio estacionário, a apresentação a seguir não faz distinção entre os conceitos de produto e hiato do produto (pois o produto natural log-linearizado é sempre zero). É imediata, porém, a extensão dos resultados aqui obtidos para o caso mais geral em que o produto natural (log-linearizado) representa choques de oferta estocásticos.

As próximas subseções descrevem o modelo, apresentando e discutindo suas principais hipóteses e equações. Para simplificar a exposição, omitem-se os detalhes da derivação dessas equações.

\subsection{Demanda}

Supõe-se um contínuo de consumidores/produtores indexados por $i \in(0,1)$. O consumidor $i$ maximiza a seguinte função de bem-estar:

$$
E_{0}\left\{\sum_{t=0}^{\infty} \beta^{t}\left[u\left(C_{t}^{i}, C_{t-1}^{i}, \tilde{M}_{t}^{i}\right)-v\left(y_{t}(i)\right)\right]\right\}
$$

onde $C_{t}^{i}$ e $C_{t-1}^{i}$ são os índices de consumo agregado (à la Dixit-Stiglitz) do consumidor $i$ nos períodos $t$ e $t-1$ respectivamente, $\tilde{M}_{t}^{i}=M_{t}^{i} / P_{t}$ representa seus saldos monetários reais no período $t, y_{t}(i)$ é a quantidade do produto diferenciado ofertado pelo consumidor $i$ no período $t, \beta \in(0,1)$ é o fator de desconto intertemporal e $E_{0}$ denota expectativas formadas racionalmente no período 0 . Vale notar que, assim como em grande parte da literatura, supõe-se que os consumidores ofertem produtos (diferenciados) diretamente. ${ }^{7}$

Como de costume, o consumidor deriva utilidade do consumo e do lazer - e, portanto, desutilidade da oferta de bens. A inclusão do argumento $C_{t-1}^{i}$ na função de utilidade instantânea $u($.$) está associada$ à hipótese de formação de hábito no consumo; supõe-se, por simplicidade, que o nível de referência para a formação de hábito seja dado pelo consumo no período anterior. ${ }^{8}$ Por sua vez, a inclusão do argumento $\tilde{M}_{t}^{i}$ reflete a ocorrência de friç̧ões nas transações, que implica que o consumidor deriva utilidade diretamente da posse de moeda, dada sua função facilitadora das transações.

As seguintes restrições são impostas às funções $u($.$) e v($.$) :$

$$
\begin{array}{cl}
u_{1}=\frac{\partial u}{\partial C_{t}}>0 ; & u_{11}=\frac{\partial^{2} u}{\partial C_{t}^{2}}<0 \\
u_{3}=\frac{\partial u}{\partial \tilde{M}_{t}}>0 ; & u_{33}=\frac{\partial^{2} u}{\partial \tilde{M}_{t}^{2}}<0
\end{array}
$$

monetária ótima deveria tentar minimizar seus desvios em torno de um nível positivo suficientemente elevado, de modo a evitar sua aproximação ao limite inferior dado por zero - reduzindo, assim, a probabilidade de ocorrerem estados da natureza em que a política ótima não fosse factível, por exigir taxas de juros negativas. Essa questão, porém, parece pouco relevante para a análise de processos de desinflação, caracterizados por níveis iniciais de taxas de juros elevados e, portanto, naturalmente distantes do limite teórico inferior dado por zero - o que explica a opção pelas friç̧ões transacionais como forma de introduzir o dilema entre estabilizar a inflação ou a taxa de juros.

${ }^{7}$ Uma abordagem alternativa, que permitiria modelar explicitamente o mercado de trabalho, seria postular que os consumidores ofertassem tipos especializados de trabalho, que seriam então contratados pelas firmas como insumos na produção dos bens diferenciados. Mas, conforme ressalta Woodford (2003, cap. 3), as duas abordagens apresentam implicações idênticas para as equações estruturais a serem derivadas. Tendo em vista que não há, no presente trabalho, interesse em analisar o mercado de trabalho, opta-se pela abordagem mais simples.

${ }^{8}$ Esta é a especificação do fenômeno de formação de hábito mais comum na literatura sobre política monetária (Amato e Laubach, 2003, Boivin e Giannoni, 2003, Woodford, 2003). 


$$
\begin{gathered}
u_{12}=\frac{\partial^{2} u}{\partial C_{t} \partial C_{t-1}}>0 \\
u_{13}=\frac{\partial^{2} u}{\partial C_{t} \partial \tilde{M}_{t}}>0 \\
v_{1}=\frac{\partial v}{\partial y_{t}}>0 ; \quad v_{11}=\frac{\partial^{2} v}{\partial y_{t}^{2}}>0
\end{gathered}
$$

As condições (4a) e (4b) correspondem às hipóteses usuais relativas à função de utilidade instantânea, suposta estritamente crescente e côncava nos argumentos $C_{t}$ e $\tilde{M}_{t}$. Nenhuma condição é imposta às derivadas de $u($.$) em relação a C_{t-1}$, pois estas não são necessárias para os resultados a serem obtidos. As condições (4e) também são convencionais, não requerendo comentários adicionais.

As hipóteses cruciais referem-se às condições (4c) e (4d). A condição (4c) reflete a não-separabilidade temporal da função utilidade associada à existência de formação de hábito, que determina que a utilidade marginal do consumo corrente deve depender positivamente do nível do consumo passado. A condição (4d), por sua vez, implica a não-separabilidade da função utilidade entre consumo e moeda, sendo a utilidade marginal do consumo (corrente) uma função positiva dos saldos monetários reais. Conforme ressalta Woodford (2003, cap. 4), é razoável supor que essa condição seja válida na presença de friç̧ões nas transações, pois os benefícios marginais da posse de moeda devem aumentar com o volume real de transações, de modo que devemos ter $u_{31}>0$ e, portanto, $u_{13}>0 .{ }^{9}$

Na formulação da restrição orçamentária do consumidor, adotam-se duas hipóteses simplificadoras: ${ }^{10}$

(i) a alocação inicial de riqueza financeira garante que todos os consumidores se defrontam com a mesma restrição orçamentária inicial;

(ii) os mercados financeiros são completos, de modo que os consumidores podem proteger-se contra seus riscos idiossincráticos - em particular, contra os riscos de renda associados à dispersão de preços entre os agentes.

Sob tais hipóteses, todos os consumidores se deparam com restrições orçamentárias intertemporais idênticas em todos os períodos e escolhem planos contingentes de consumo e detenção de saldos monetários também idênticos - sendo possível, portanto, trabalhar com a noção de um 'consumidor representativo' e omitir os sobrescritos $i$ nos termos $C_{t}^{i}$ e $\tilde{M}_{t}^{i}$.

Em cada período $t$, o consumidor está sujeito à seguinte restrição orçamentária:

$$
M_{t}+B_{t}+\int_{0}^{1} p_{t}(i) c_{t}(i) d i \leq P_{t} Y_{t}+T_{t}+M_{t-1} R_{t-1}^{m}+B_{t-1} R_{t-1}
$$

onde $M_{t}$ e $B_{t}$ são, respectivamente, os estoques nominais de moeda e títulos do governo detidos ao final do período $t, R_{t-1}$ é a taxa de juros nominal (bruta) dos títulos entre os períodos $t-1 \mathrm{e} t, R_{t-1}^{m}$ é a taxa de juros nominal (bruta) incidente sobre os saldos monetários detidos ao final do período $t-1, P_{t}$ é o índice de preço agregado, $Y_{t}$ é o índice agregado das quantidades ofertadas dos bens diferenciados, $T_{t}$ é o valor nominal das transferências líquidas recebidas do governo e o termo $\int_{0}^{1} p_{t}(i) c_{t}(i) d i$ designa o dispêndio nominal total no consumo dos bens diferenciados.

O problema do consumidor é escolher, para $t \geq 0$, trajetórias do consumo, $\left\{C_{t}\right\}$, e dos estoques reais de moeda e títulos, $\left\{\tilde{M}_{t}\right\}$ e $\left\{\tilde{B}_{t}\right\}$, de modo a maximizar (3) sujeito a (5). Conforme mostra o Apêndice $A$, a solução desse problema fornece o bloco de demanda do modelo:

\footnotetext{
${ }^{9}$ No contexto de um modelo simples em que a realização de compras de bens requer tempo, Walsh (1998, cap. 3) mostra que para que essa condição sejá válida, é suficiente que o aumento do consumo eleve a produtividade marginal da moeda na redução do tempo utilizado nas compras.

${ }^{10}$ Essas hipóteses são usuais na literatura. Para uma discussão mais detalhada de suas implicações, ver Woodford (2003, cap. 3).
} 


$$
\begin{aligned}
y_{t}= & \delta_{1} y_{t-1}+\delta_{2} E_{t} y_{t+1}+\delta_{3} E_{t} y_{t+2}+\delta_{4}\left(r_{t}-E_{t} \pi_{t+1}\right) \\
& +\delta_{5} m_{t}+\delta_{6} E_{t} m_{t+1}+\delta_{7} E_{t} m_{t+2} \\
m_{t}= & \eta_{1} y_{t}+\eta_{2} y_{t-1}+\eta_{3} E_{t} y_{t+1}+\eta_{4} r_{t}+\eta_{5} E_{t} m_{t+1}
\end{aligned}
$$

onde $y_{t}, r_{t}, \pi_{t}$ e $m_{t}$ representam os desvios do produto, da taxa de juros (bruta), da taxa de inflação (bruta) e do estoque real de moeda em relação a seus valores no equilíbrio estacionário com inflação zero. Os coeficientes dessas equações são funções dos parâmetros estruturais da economia, conforme especificado no Apêndice.

As equações (6) e (7) podem ser interpretadas como equações IS e LM intertemporais. Vale destacar as diferenças entre a curva IS acima e a IS do modelo novo-keynesiano básico (equação (1)):

(i) a presença dos termos adicionais $y_{t+2}$ e $y_{t-1}$ - este último sendo responsável pela 'inércia' do produto;

(ii) a presença dos saldos monetários reais (correntes e futuros), que implicam a ocorrência de 'efeitos de saldos reais' na demanda.

Essas diferenças devem-se às hipóteses de formação de hábito e fricções transacionais adotadas no trabalho; evidentemente, na ausência desses fenômenos, a equação (6) se reduz à IS do modelo básico. ${ }^{11}$

Outra diferença importante em relação ao modelo básico diz respeito ao papel da curva LM. Na análise padrão, essa equação limita-se a determinar a quantidade de moeda implícita em uma regra monetária definida em termos do controle da taxa de juros - revelando-se, assim, desnecessária para determinar a evolução do produto e das taxas de inflação e juros. Aqui, não é em geral possível desconsiderar a curva LM, devido aos efeitos cruzados decorrentes da não separabilidade da função utilidade entre consumo e moeda.

\subsection{Oferta}

No seu papel de ofertante, cada agente deve escolher o preço a ser cobrado por seu produto em cada momento, $p_{t}(i)$; a esse preço, ele deve satisfazer toda a demanda pelo produto, dada por

$$
y_{t}(i)=\left(\frac{p_{t}(i)}{P_{t}}\right)^{-\theta} Y_{t}
$$

onde $\theta>1$. Tendo em vista que os ofertantes operam em um ambiente de competição monopolística, eles tomam como dados os valores das variáveis agregadas $P_{t}$ e $Y_{t}$ ao escolher seus preços.

A escolha de $p_{t}(i)$ deve procurar maximizar a contribuição líquida da receita de vendas para a utilidade do agente, descontada a desutilidade associada à oferta do produto, em conformidade com a função de bem-estar (3). Caso houvesse perfeita flexibilidade de preços, o problema do ofertante seria simplesmente maximizar, em cada período $t$, o 'lucro instantâneo'

$$
\begin{aligned}
\Gamma_{t} & =\left(\frac{\Lambda_{t}}{P_{t}}\right) p_{t}(i) y_{t}(i)-v\left(y_{t}(i)\right) \\
& =\Lambda_{t}\left(\frac{p_{t}(i)}{P_{t}}\right)^{1-\theta} Y_{t}-v\left[\left(\frac{p_{t}(i)}{P_{t}}\right)^{-\theta} Y_{t}\right]
\end{aligned}
$$

\footnotetext{
$\overline{{ }^{11} \mathrm{Na} \text { ausência de formação de hábito, temos }} \delta_{1}=\delta_{3}=0$ e $\delta_{2}=1$; e na ausência de fricções transacionais (ou sob separabilidade aditiva da função utilidade entre consumo e saldos reais), $\delta_{5}=\delta_{6}=\delta_{7}=0$.
} 
onde $\Lambda_{t} / P_{t}$ denota a utilidade marginal da renda nominal e na segunda linha usou-se a equação de demanda (8). Supõe-se, porém, a existência de rigidez nominal de preços, de modo que nem todos os ofertantes têm a possibilidade de escolher preços ótimos em cada momento. Em particular, adotase a versão discreta do mecanismo de Calvo (1983) modificada pela presença de indexação, conforme Christiano et alii (2001): em cada período $t$, uma fração $1-\alpha$ dos ofertantes, sorteada aleatoriamente, tem a possibilidade de escolher um novo preço 'ótimo', enquanto a fração $\alpha$ restante deve cobrar o preço vigente no período $t-1$, ajustado por uma parcela $\gamma$ da inflação observada em $t-1$ (isto é, multiplicado por $\Pi_{t-1}^{\gamma}$, onde $\Pi_{t-1}$ é a taxa de inflação bruta entre $t-2$ e $t-1$ ), independentemente do tempo transcorrido desde a última vez em que tais ofertantes foram sorteados para otimizar seus preços. Os ofertantes sorteados no período t devem, portanto, escolher um preço $P_{t}^{*}$ de modo a maximizar o valor esperado do fluxo descontado de lucros instantâneos:

$$
\begin{array}{r}
E_{t} \sum_{j=0}^{\infty}(\alpha \beta)^{j}\left\{\Lambda_{t+j}\left[\frac{P_{t}^{*}}{P_{t+j}}\left(\frac{P_{t+j-1}}{P_{t-1}}\right)^{\gamma}\right]^{1-\theta} Y_{t+j}\right. \\
\left.-v\left[\left[\frac{P_{t}^{*}}{P_{t+j}}\left(\frac{P_{t+j-1}}{P_{t-1}}\right)^{\gamma}\right]^{-\theta} Y_{t+j}\right]\right\}
\end{array}
$$

onde a taxa de desconto utilizada nesse cálculo é $\alpha \beta$, em vez de simplesmente $\beta$, tendo em vista que a probabilidade do preço escolhido na data $t$ continuar valendo no período $t+j$ é igual a $\alpha^{j}$.

Conforme mostra o Apêndice $B$, a condição de primeira ordem desse problema conduz, após algumas manipulações algébricas, à equação de oferta agregada do modelo:

$$
\begin{aligned}
\pi_{t}-\gamma \pi_{t-1}= & \mu_{1} y_{t}+\mu_{2} y_{t-1}+\mu_{3} E_{t} y_{t+1}+\mu_{4} m_{t}+\mu_{5} E_{t} m_{t+1} \\
& +\beta\left(E_{t} \pi_{t+1}-\gamma \pi_{t}\right)
\end{aligned}
$$

onde os coeficientes são funções dos parâmetros estruturais da economia, conforme explicitado no Apêndice.

A comparação entre a equação de oferta (11) e a curva de Phillips do modelo novo-keynesiano básico (equação (2)) aponta duas diferenças básicas:

(i) a presença dos termos adicionais $y_{t+1}$ e $y_{t-1}$;

(ii) a presença dos saldos monetários reais (correntes e futuros), que refletem a ocorrência de 'efeitos de saldos reais' na demanda.

Essas diferenças devem-se às hipóteses de formação de hábito e friç̧ões transacionais aqui adotadas; evidentemente, na ausência desses fenômenos, a equação se reduziria à equação 2 .

A equação (11) compõe, junto com (6) e (7), o sistema de equações estruturais do modelo.

\subsection{Bem-Estar}

Uma das principais vantagens de trabalhar com um modelo microfundamentado diz respeito ao fato de que a função utilidade do agente representativo fornece um critério natural para a avaliação de políticas econômicas alternativas. Seguindo o método originalmente proposto por Rotemberg e Woodford (1997) e discutido em detalhe em Woodford (2003, cap. 6), pode-se derivar o critério de bemestar relevante a partir de uma aproximação de Taylor de segunda ordem da utilidade esperada do agente representativo: 


$$
W=E\left\{\sum_{t=0}^{\infty} \beta^{t} U_{t}\right\}
$$

onde

$$
U_{t}=u\left(Y_{t}, Y_{t-1}, \tilde{M}_{t}\right)-\int_{0}^{1} v\left(y_{t}(i)\right) d i
$$

Conforme mostra o Apêndice $C$, o problema de maximizar o critério de bem-estar assim derivado é equivalente ao problema de minimizar

$$
\sum_{t=0}^{\infty} \beta^{t}\left[\left(\pi_{t}-\gamma \pi_{t-1}\right)^{2}+\lambda_{y}\left(y_{t}-\zeta y_{t-1}\right)^{2}+\lambda_{r}\left(r_{t}-r^{*}\right)^{2}\right]
$$

que é a 'função perda' a ser usada na avaliação das regras 'arbitrárias' de desinflação consideradas na Seção 3 e na derivação da regra ótima na Seção 4.

\subsection{Forma Funcional e Parametrização}

Até o momento, todos os resultados foram obtidos para uma função de utilidade instantânea genérica $u($.$) . Nas simulações a serem realizadas nas próximas seções, utiliza-se uma forma funcional$ específica para essa função, que satisfaça as condições (4a)-(4d):

$$
u\left(C_{t}, C_{t-1}, \tilde{M}_{t}\right)=Z\left[\left(\frac{C_{t}}{C_{t-1}^{h}}\right)^{1-\sigma}\right]^{d}\left[\left(C_{t} \varphi\left(\tilde{M}_{t}\right)\right)^{1-\tau}\right]^{1-d}
$$

onde

$$
\begin{array}{cc}
\sigma>1 & 0 \leq h \leq 1 \\
0<\tau<1 & 0 \leq d \leq 1 \\
\varphi^{\prime}\left(\tilde{M}_{t}\right)>0 & \varphi^{\prime \prime}\left(\tilde{M}_{t}\right)<0
\end{array}
$$

Opta-se por não especificar a forma da função $\varphi\left(\tilde{M}_{t}\right)$ pois, ao adotar a hipótese de saciedade em termos de saldos monetários, essa função deve simplesmente satisfazer $\varphi^{\prime}\left(\tilde{M}_{t}\right) \longrightarrow 0$.

Apesar de pouco convencional, a especificação (14) é interessante por englobar como casos particulares a forma funcional multiplicativa comum na literatura sobre formação de hábito (que ocorre quando $d=1$ e $Z=1 /(1-\sigma))$ e a forma funcional tradicional em modelos com moeda na função de utilidade (que ocorre quando $d=0, Z=1 /(1-\tau)$ e $\left.\varphi\left(\tilde{M}_{t}\right)=\tilde{M}_{t}^{b}\right) .{ }^{12}$ O uso dessa especificação facilita, assim, a tarefa de calibragem do modelo a partir dos parâmetros usados em estudos anteriores.

Com base nas estimativas e calibragens para a economia norte-americana apresentadas em Rotemberg e Woodford (1997), Ireland (2001), Amato e Laubach (2003), Boivin e Giannoni (2003) e Woodford (2003), adota-se como 'parametrização básica' para os parâmetros estruturais os valores constantes da Tabela 1. Evidentemente, serão consideradas parametrizações alternativas para tais parâmetros, quando relevante.

Cabe notar que a hipótese de que a economia se encontra suficientemente próxima do nível de saciedade em termos de saldos monetários implica as seguintes restrições sobre os parâmetros da IS e da curva de Phillips derivadas acima:

\footnotetext{
${ }^{12}$ Alguns artigos que utilizam essa forma funcional multiplicativa para retratar a formação de hábito são Fuhrer (2000) e Amato e Laubach (2003). Para uma discussão da abordagem da 'moeda na função de utilidade' e exemplos de formas funcionais, ver Walsh (1998, cap.2).
} 
Tabela 1: Parametrização básica

\begin{tabular}{cccc}
\hline Parâmetro & Valor & Parâmetro & Valor \\
\hline$\beta$ & $0.96^{1 / 4}$ & $\sigma$ & 1.5 \\
\hline$\alpha$ & 0.75 & $\omega$ & 0.47 \\
\hline$\theta$ & 8 & $\gamma$ & 0.9 \\
\hline$\nu$ & 0.36 & $h$ & 0.5 \\
\hline$\eta_{r}$ & 7.8 & $d$ & 0.6 \\
\hline$\tau$ & 0.2 & &
\end{tabular}

$$
\begin{array}{ll}
\delta_{5} \longrightarrow 0 & \mu_{4} \longrightarrow 0 \\
\delta_{6} \longrightarrow 0 & \mu_{5} \longrightarrow 0 \\
\delta_{7} \longrightarrow 0 &
\end{array}
$$

de modo que, para efeito dos exercícios a seguir, será possível efetivamente desconsiderar a existência dos efeitos dos saldos reais nessas equações e trabalhar com elas como se não houvesse friç̧ões monetárias. Como mencionado anteriormente, esse procedimento parece razoável à luz dos resultados empíricos de, inter alia, Ireland (2001) e Woodford (2003, cap. 4), que estimam, para a economia dos EUA, efeitos dos saldos reais pouco significativos do ponto de vista quantitativo. Para os propósitos deste artigo, a função da introdução da moeda na função de utilidade se resume, assim, a gerar um motivo para o objetivo de estabilização da taxa de juros.

\section{DESINFLAÇÃO SOB REGRAS 'ARBITRÁRIAS’ DE POLÍTICA MONETÁRIA}

Esta seção analisa, no contexto do modelo desenvolvido acima, as características de políticas de desinflação implementadas a partir de regras 'arbitrárias' de política monetária - isto é, regras que não são especificadas com base em algum critério de otimização.

Supõe-se que a economia se encontre inicialmente em equilíbrio a uma taxa de inflação positiva mas moderada, de modo a garantir a validade das equações log-linearizadas em torno do estado estacionário com inflação zero. Em determinado período (definido como o período 0) as autoridades monetárias anunciam, de modo inesperado e crível, a adoção de uma nova regra de política monetária, que deverá conduzir a economia ao equilíbrio com inflação zero.

0 instrumento de política monetária é a taxa de juros básica, $r_{t}$. A autoridade monetária anuncia que, a partir do período 0 , a política de juros será conduzida com base na seguinte regra (loglinearizada):

$$
\begin{aligned}
r_{t} & =\tilde{r}_{t}+\pi_{t}^{*}+\psi\left(\pi_{t}-\pi_{t}^{*}\right) \\
\pi_{t}^{*} & =a \pi_{t-1}^{*}
\end{aligned}
$$

onde $\tilde{r}_{t}$ é a taxa real de juros (suposta constante, por simplicidade), $\pi_{t}^{*}$ é a 'meta de inflação implícita' e $a$ e $\psi$ são parâmetros tais que $0 \leq a<1$ e $\psi>1$. Segundo essa regra:

(i) a meta implícita deve convergir assintoticamente para o nível de inflação zero, a uma velocidade determinada pela magnitude do coeficiente $a$;

(ii) a taxa de juros nominal deve ser ajustada em função dos desvios entre a inflação observada e a meta implícita, sendo a sensibilidade a tais desvios dada pelo parametro $\psi$. 
Evidentemente, (16a) poderia conter termos adicionais - relativos, por exemplo, ao produto, como na regra popularizada por Taylor (1993) -; mas se, por um lado, tais complicações devem ser fundamentais na formulação de uma regra de reação a distúrbios estocásticos, por outro lado sua utilidade é questionável quando o objetivo é determinar trajetórias determinísticas para as variáveis do sistema. ${ }^{13}$

A partir do período 0 , o funcionamento da economia passa a ser descrito pelo sistema de equações composto por (6), (11) e (16a)-(16b). Sujeito à restrição $\psi>1$ (necessária para satisfazer o 'princípio de Taylor'), o sistema é localmente determinado para parametrizações convencionais das equações estruturais - e, em particular, para a parametrização 'básica' apresentada na seção anterior. O sistema é colocado na forma padrão de Blanchard e Kahn (1980) e resolvido através dos procedimentos numéricos descritos em King e Watson (1998). ${ }^{14}$

A seguir, discutem-se várias simulações do modelo. Em todas as simulações, parte-se de uma inflação de $10 \%$ ao ano e taxa de juros nominal de aproximadamente $14,5 \%$ a.a., correspondente a uma taxa real de $4 \% .{ }^{15}$ Pode-se argumentar que a magnitude da taxa de inflação inicial considerada seja excessivamente elevada, comprometendo os resultados obtidos a partir de um modelo log-linearizado em torno do equilíbrio com inflação zero; mas esse procedimento encontra respaldo em vários trabalhos anteriores. ${ }^{16}$ Mais importante do que isso, porém, é o fato de que o nível de $10 \%$ encontra-se dentro dos limites necessários para garantir a existência dos preços ótimos de equilíbrio definidos no problema do ofertante e, em última instância, a validade do modelo. ${ }^{17}$

O valor inicial do produto depende negativamente do grau de indexação dos preços não-otimizados. De fato, dado que a economia encontra-se inicialmente em um equilíbrio estacionário do modelo loglinearizado, o valor do produto no período - 1 pode ser calculado pela curva de Phillips como:

$$
\bar{y}=\left[\frac{(1-\gamma)(1-\beta)}{\mu_{1}+\mu_{2}+\mu_{3}}\right] \bar{\pi}
$$

onde $\bar{y}$ e $\bar{\pi}$ denotam os valores constantes de $y$ e $\pi$ no equilibrio inicial. Nos gráficos apresentados a seguir, o valor do produto no equilibrio final (com inflação zero) é normalizado em 100.

Vale ressaltar uma implicação óbvia, mas importante, da relação de equilíbrio (17). Tal relação vale, evidentemente, para ambos equilíbrios inicial e final; dado que a taxa de inflação inicial é maior do que a inflação final, o mesmo deve ocorrer para o produto. Assim, o experimento de desinflação aqui analisado produz necessariamente, em algum momento, um efeito 'recessivo'. ${ }^{18}$ Resta investigar a magnitude e persistência dessa recessão.

\footnotetext{
${ }^{13}$ De fato, será visto na Seção 4 que a regra simples proposta acima consegue, sob certas parametrizações, aproximar-se arbitrariamente da regra ótima pela escolha adequada dos coeficientes $a$ e $\psi$.

${ }^{14}$ Para resolver e analisar o modelo, usa-se o conjunto de rotinas 'Reds-Solds' (na página de Michael Woodford na internet:

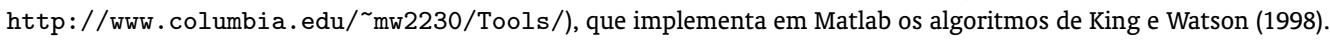

${ }^{15} \mathrm{O}$ modelo foi calibrado em base trimestral, mas todos os gráficos mostram taxas anualizadas, de modo a facilitar a interpretação dos resultados.

${ }^{16}$ Por exemplo, Erceg e Levin (2003) e Woodford (2003).

${ }^{17}$ Conforme observado por Ascari (2004) e Bakhshi et alii (2003), o equilíbrio de estado estacionário de um modelo à la Calvo só está definido (pela convergência dos somatórios na equação do preço relativo ótimo) para taxas de inflação relativamente baixas. No presente contexto, porém, pode-se mostrar que esse equilíbrio de fato existe para taxas de inflação de $10 \%$ a.a ou menos.

${ }^{18}$ Esse resultado depende crucialmente do fato de que estamos aproximando as variáveis sempre em torno do mesmo equilíbrio de estado estacionário do modelo exato - o equilíbrio com inflação zero. Esse procedimento justifica-se pela interpretação do equilíbrio inicial do modelo log-linearizado como um desvio em relação ao equilíbrio (com inflação zero) do modelo exato, que parece razoável no caso de 'pequenas desinflações'. Uma abordagem alternativa, mais adequada para 'grandes desinflações', seria interpretar a desinflação como a passagem de um equilíbrio de estado estacionário (com inflação positiva) do modelo exato para outro equilíbrio de estado estacionário (com inflação zero) do modelo exato, conforme feito, por exemplo, por Ireland (1995) e Calvo et alii (2003). Nesse caso, o processo de desinflação poderia levar a um ganho de produto, tendo em vista que o nível de produto de equilíbrio do modelo exato depende negativamente da taxa de inflação de equilíbrio.
} 
Figura 1: Trajetórias sob a regra 'arbitrária' de desinflação $(a=0.841, \psi=1.25, h=0.5)$
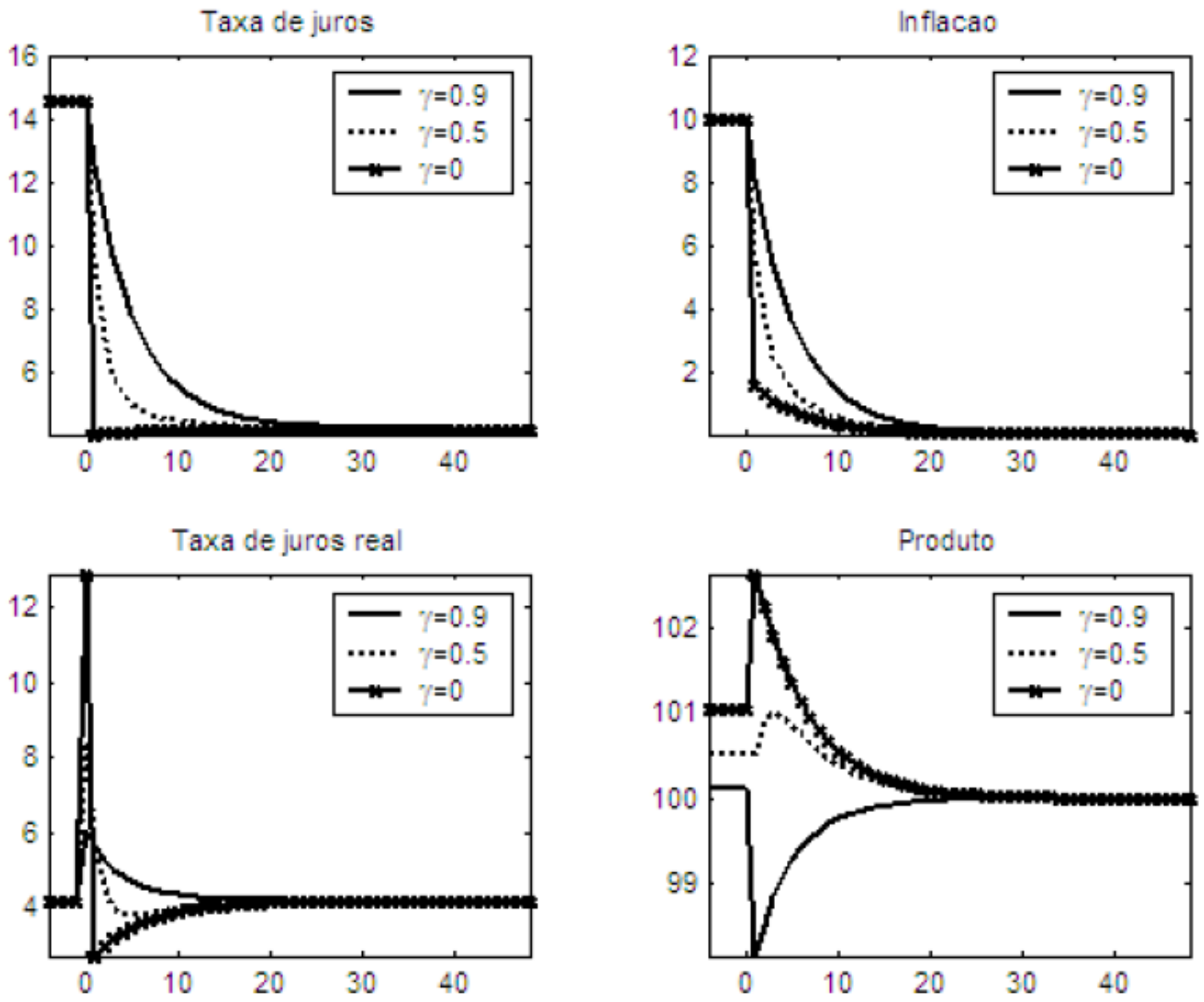

Obs.: Apresentam-se as trajetórias da taxa de juros nominal e real, inflação e produto sob três cenários distintos, caracterizados por diferentes graus de indexação: $\gamma=0.9$ (linha sólida), $\gamma=0.5$ (linha pontilhada) e $\gamma=0$ (linha sólida com marcadores). Em todos os casos, supõe-se $a=0.841, \psi=1.25$ e $h=0.5$.

A Figura 1 apresenta as trajetórias das variáveis endógenas do sistema sob a regra de desinflação (16a)-(16b), usando a parametrização 'básica' e os seguintes valores para os parâmetros da regra monetária: $a=0.841$ e $\psi=1.25$. O valor do parâmetro $a$ foi escolhido de modo que a meta de inflação implícita após 4 períodos (ou seja, 1 ano) corresponda a 50\% da taxa de inflação inicial; o valor de $\psi$, por sua vez, corresponde ao menor valor considerado que satisfaça o 'princípio de Taylor'. O grau de formação de hábito na economia é dado por $h=0.5$, e consideram-se três situações distintas no que se refere ao grau de indexação dos preços não-otimizados: $\gamma=0, \gamma=0.5$ e $\gamma=0.9$.

Observa-se que, como seria razoável esperar, a velocidade de convergência da inflação para o nível zero varia inversamente com o grau de indexação. Dada a mesma trajetória para a meta implícita (definida pelo parâmetro $a$ ), diferentes graus de indexação implicam diferentes requisitos de tempo para reduzir a inflação a níveis 'aceitáveis'. Por exemplo, para que a inflação seja reduzida para $2 \%$ são necessários cerca de 8 períodos (dois anos) no caso $\gamma=0.9$, entre 3 e 4 períodos (menos de um ano) no caso $\gamma=0.5$ e apenas 1 período (um trimestre) na ausência de indexação. 
O resultado mais interessante diz respeito à trajetória do produto. Em todos os casos considerados, o nível final do produto está evidentemente abaixo do nível inicial, conforme discutido anteriormente; e, no caso de indexação 'elevada' $(\gamma=0.9)$, a adoção da nova política gera uma forte recessão inicial, em conformidade com os fatos estilizados dos processos de desinflação. Entretanto, nos casos em que o grau de indexação é 'baixo' ou nulo, o início do processo é caracterizado por um boom, seguido de uma lenta convergência para o novo equilíbrio, o que se contrapõe aos fatos estilizados. Simulações adicionais, não reportadas por razões de espaço, indicam que o grau de indexação 'crítico' abaixo do qual ocorre um boom é aproximadamente 0.5 .

A Figura 2 compara o valor da perda de bem-estar (13) sob diferentes magnitudes da velocidade da moeda $\nu=(\bar{Y} / \bar{M})$ - que assume os valores $0.36,1$ e 1000 nos gráficos da primeira, segunda e terceira coluna, respectivamente - e do grau de indexação da economia $\gamma$ - que assume os valores $0.9,0.5$ e 0 nos gráficos da primeira, segunda e terceira linha, respectivamente -, para regras alternativas da classe (16a)-(16b), diferenciadas pelos valores dos coeficientes $a$ e $\psi$ - sendo considerados três valores para 0 parâmetro $\psi(1.25,5$ e 20$)$ e um contínuo de valores para $a$ entre 0 e 1 . Os principais resultados são:

(i) em geral, as regras $\operatorname{com} \psi$ baixo dominam as regras $\operatorname{com} \psi$ mais alto;

(ii) quando o grau de indexação é 'baixo' ou nulo, parece preferível escolher o menor valor possível para $a$ (ou seja, uma desinflação mais rápida);

(iii) quando o grau de indexação é 'alto', a preferência por uma estratégia de desinflação 'rápida' ou 'lenta' depende do valor de $\nu$ : quanto maior o valor desse parâmetro, maior parece ser o $a$ 'ótimo' - e, portanto, mais lenta deve ser a desinflação.

Os resultados (ii) e (iii) estão relacionados à possível existência de um dilema entre os objetivos de estabilização da inflação e dos juros. Em economias com alto grau de indexação, esse dilema existe e é significativo: por um lado, dado que o que importa para o bem-estar do consumidor é a quase-diferença na taxa de inflação $\left(\pi_{t}-\gamma \pi_{t-1}\right)$, e não seu valor absoluto, seria ótimo desinflar lentamente; por outro lado, dado que na presença de friç̧ões monetárias o ideal é seguir a 'regra de Friedman', seria ótimo desinflar rapidamente, pois isso permitiria reduzir rapidamente, para níveis próximos de zero, também a taxa de juros. Esse último efeito é mais significativo quando a velocidade da moeda é baixa (pois nesse caso o coeficiente $\lambda_{r}$ da função perda aumenta), o que explica o resultado (iii) acima. Em economias sem indexação (ou com indexação baixa), por sua vez, o bem-estar do consumidor representativo aumenta com a estabilização do nível da inflação, de modo que não há qualquer dilema: a melhor política deve ser desinflar rapidamente, de modo a trazer ambas as taxas de inflação e de juros para níveis mais baixos.

Até o momento, todos os resultados foram obtidos supondo um grau constante de formação de hábito $(h=0.5)$. A Figura 3 mostra como mudam as trajetórias das variáveis endógenas quando o grau de formação de hábito varia. Com o objetivo de ressaltar ao máximo esse efeito, a figura apresenta as diferenças (absolutas) nos valores de cada variável ao longo do tempo sob duas hipóteses extremas: $h=1$ e $h=0$; mais especificamente, os valores nos gráficos referem-se a $\Delta_{z}(h)=z(h=1)-z(h=$ 0 ), onde $z(h=j)$ é o valor da variável $z$ quando $h=j$. Observa-se que o efeito da formação de hábito é quantitativamente pouco significativo no caso das taxas de juros e inflação, mas não desprezível no caso do produto, cujas variações chega a ampliar em mais de $0,5 \%$.

Em resumo, os resultados desta seção sugerem que, sob uma regra simples de desinflação:

i. A magnitude da recessão inicial associada ao processo de desinflação depende positivamente do grau de indexação da economia - sendo que, para níveis de indexação suficientemente baixos, pode ocorrer inicialmente um boom, em vez de recessão;

ii. As variações (positivas ou negativas) do produto são amplificadas quando o grau de formação de hábito é elevado; 
Figura 2: Perda de bem-estar sob regras ‘arbitrárias' de desinflação
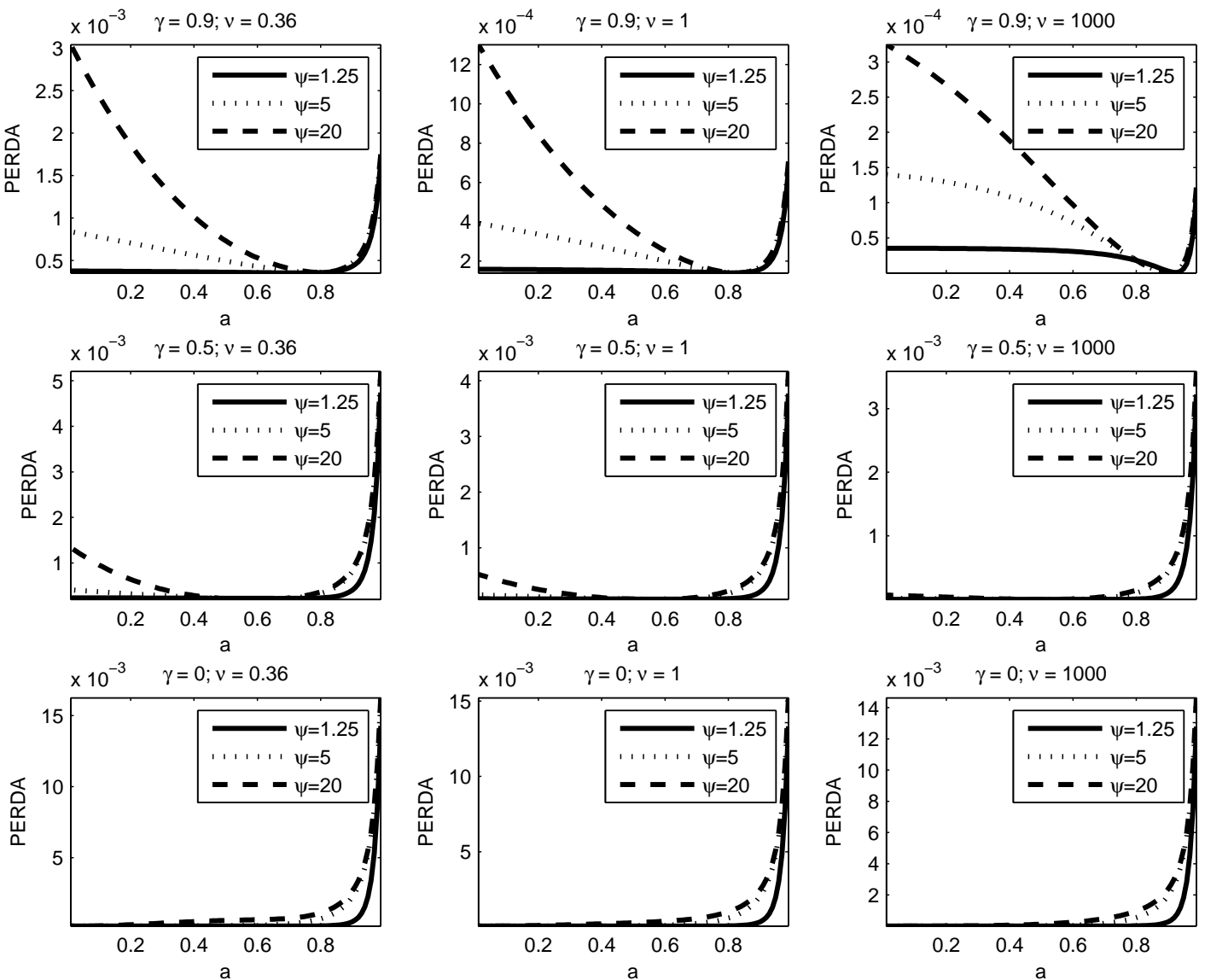

Obs.: A perda de bem-estar é calculada para um contínuo de valores do coeficiente $a$ da regra monetária, sob diferentes magnitudes da velocidade da moeda $\nu=(\bar{Y} / \bar{M})$ - que assume os valores $0.36,1$ e 1000 nos gráficos da primeira, segunda e terceira coluna, respectivamente -, do grau de indexação da economia $\gamma$ - que assume os valores $0.9,0.5$ e 0 nos gráficos da primeira, segunda e terceira linha, respectivamente -, e do coeficiente $\psi$ - sendo $\psi=1.25$ nas linhas sólidas, $\psi=5$ nas linhas pontilhadas e $\psi=20$ nas linhas tracejadas.

iii. A velocidade ótima da desinflação depende negativamente do grau de indexação na economia;

iv. Em economias altamente indexadas, a velocidade ótima da desinflação depende negativamente da velocidade da moeda (ou, em termos mais gerais, positivamente da magnitude das friç̧ões monetárias).

\section{DESINFLAÇÃO ÓTIMA}

Esta seção analisa as propriedades da política ótima de desinflação sob parametrizações alternativas das equações estruturais do modelo. Novamente, supõe-se que as autoridades monetárias anunciem na data 0 , de forma inesperada e crível, a adoção permanente de uma regra de política monetária visando conduzir a economia do equilíbrio inicial com inflação 'alta' ao novo equilíbrio com inflação 'baixa'. 
Figura 3: Efeito da formação de hábito (diferenças nas trajetórias sob $h=1$ e $h=0$ )
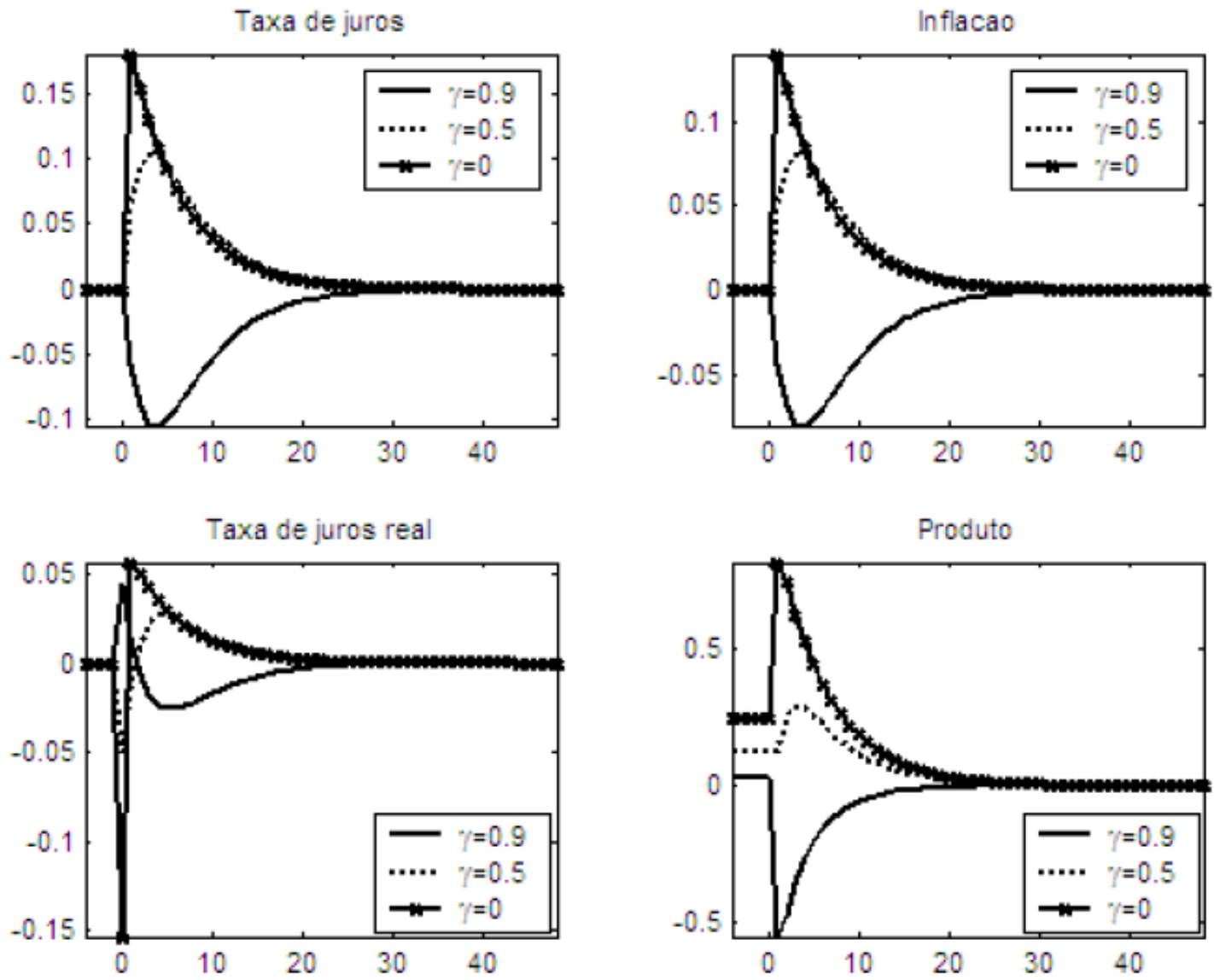

Obs.: Apresentam-se as diferenças nas trajetórias sob $h=1$ e $h=0$, para três cenários distintos, caracterizados por diferentes graus de indexação: $\gamma=0.9$ (linha sólida), $\gamma=0.5$ (linha pontilhada) e $\gamma=0$ (linha sólida com marcadores). Em todos os casos, supõe-se $a=0.841, \psi=1.25$ e $h=0.5$.

A política ótima de desinflação é derivada a partir da minimização da função de perda (13), sujeita às restrições impostas pelas equações estruturais (6) e (11). Conforme detalhado no Apêndice $D$, a solução desse problema dá origem à seguinte 'regra instrumental implícita', na terminologia de Woodford (2003):

$$
\tilde{\pi}_{t}+\Phi_{y}(L) \tilde{y}_{t}=\Phi_{r}(L)\left(r_{t}-r^{*}\right)
$$

onde

$$
\begin{aligned}
\Phi_{y}(L) & =\lambda_{y}\left[\frac{\left(1-\beta \zeta L^{-1}\right)(1-L)}{\mu_{1}+\beta \mu_{2} L^{-1}+\beta^{-1} \mu_{3}}\right] \\
\Phi_{r}(L) & =\lambda_{r}\left[\frac{\left(1+\beta \delta_{1} L^{-1}+\beta^{-1} \delta_{2} L+\beta^{-2} \delta_{3} L^{2}\right)(1-L)}{\delta_{4}\left(\mu_{1}+\beta \mu_{2} L^{-1}+\beta^{-1} \mu_{3}\right)}-\frac{\beta^{-1} L}{1-\beta \gamma L^{-1}}\right]
\end{aligned}
$$

Essa regra resume o comportamento a ser adotado pelas autoridades monetárias em todos os períodos $t \geq 2$; para os períodos $t=0,1$ a regra deve ser modificada em função de certas condições iniciais 
especificadas no Apêndice. ${ }^{19}$ É interessante observar que a regra (18) contém como casos particulares algumas das regras ótimas derivadas por Woodford (2003) e Giannoni e Woodford (2004), sob especificações alternativas das equações estruturais. Em particular, supondo $\zeta=0, \gamma=0$ e $\lambda_{r}=0$, a regra acima se reduz a

$$
\pi_{t}=-\frac{\lambda_{y}}{\tilde{\kappa}\left(\omega-\epsilon_{11}\right)}\left(y_{t}-y_{t-1}\right)
$$

que é a regra ótima no modelo novo-keynesiano básico na ausência de friç̧ões monetárias.

Sob a regra ótima, os valores da inflação, da taxa de juros e do produto no equilíbrio em estado estacionário (do modelo log-linearizado) são dados por:

$$
\begin{aligned}
\bar{\pi} & =\bar{r}=\left[\frac{\lambda_{r}}{\beta(1-\beta \gamma)(1-\gamma)+\lambda_{r}}\right] \\
\bar{y} & =\left[\frac{(1-\gamma)(1-\beta)}{\mu_{1}+\mu_{2}+\mu_{3}}\right] \bar{\pi}
\end{aligned}
$$

onde a expressão para $\bar{y}$ acima é a mesma apresentada anteriormente (equação (17)). Uma implicação importante de (20) é que, em geral, $\bar{\pi}<0$, de modo que o plano ótimo envolve deflação, e não inflação zero - que só deve ocorrer se $\lambda_{r}=0$ (fricções monetárias irrelevantes) ou $r^{*}=0$ (taxa nominal de juros ótima igual à taxa real no equilíbrio estacionário com inflação zero). Por outro lado, conforme observado por Woodford (2003, cap. 7), essa taxa de deflação é geralmente menos pronunciada do que a deflação preconizada pela regra de Friedman, que no presente contexto requer $\bar{\pi}=r^{*}$; claramente, essa condição se verificaria apenas se houvesse plena indexação na economia $(\gamma=1)$.

A Figura 4 apresenta as trajetórias das variáveis endógenas sob a regra ótima, para diferentes valores da velocidade da moeda $\nu$ e do grau de indexação da economia $\gamma$. Cabe notar que o equilíbrio inicial é o mesmo considerado na seção anterior; isto é, inflação de 10\%, taxa de juros de $14,5 \%$ e nível de produto dado por (21). Algumas observações merecem destaque. Primeiro, a desinflação ótima não gera um boom de atividade em nenhum dos casos considerados, ao contrário do que ocorre sob políticas 'arbitrárias' de desinflação. Observa-se, de fato, recessões significativas em todos os casos (principalmente quando a indexação é elevada) e trajetórias do produto em forma de ' $U$ ' invertido, em conformidade com os fatos estilizados mencionados anteriormente. Segundo, quando as friç̧ões monetárias são irrelevantes (isto é, no caso $\nu=1000$ ) e não há indexação, a desinflação é imediata e apresenta custos mínimos em termos de perda de produto; com indexação, a convergência para a inflação zero é apenas gradual. Terceiro, quando as fricções monetárias são relevantes (isto é, nos casos $\nu=0.36$ e $\nu=1$ ), a taxa de inflação converge para valores negativos, em vez de zero - conforme previsto pela expressão (20). Por fim, a evolução da taxa de juros mostra-se não-monotônica, caracterizando-se por forte queda inicial, seguida de elevação e gradual convergência para o novo equilíbrio. Esse padrão parece estar associado ao princípio geral de que, sob uma política ótima com comprometimento adotada na data 0 , as autoridades monetárias podem partir de condições iniciais que, por definição, não afetam as expectativas formadas antes dessa data - obtendo, assim um 'ganho' instantâneo. ${ }^{20}$ No caso em questão, a forte redução inicial da taxa de juros visa:

(i) reduzir ao máximo as distorções causadas pela existência de friç̧ões monetárias na data 0; e, ao mesmo tempo,

(ii) criar expectativas de aumento da taxa de juros (nominal e real) nos períodos subsequentes, gerando recessão e abrindo caminho para a redução da taxa de inflação.

\footnotetext{
${ }^{19}$ Caso a política ótima fosse derivada com base na chamada 'perspectiva atemporal', essa regra valeria inalterada para todos os períodos $t \geq 0$ (Woodford, 2003).

${ }^{20}$ Ver, por exemplo, a discussão em Woodford (2003, caps. 6 e 7).
} 
Figura 4: Trajetórias sob a regra ótima de desinflação $\left(r^{*}<0\right)$
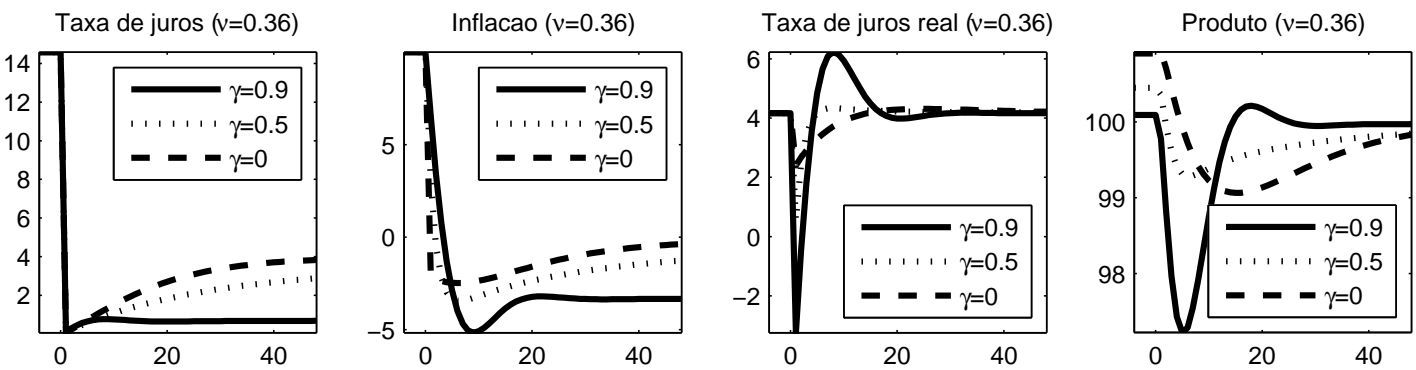

Taxa de juros $(v=1)$
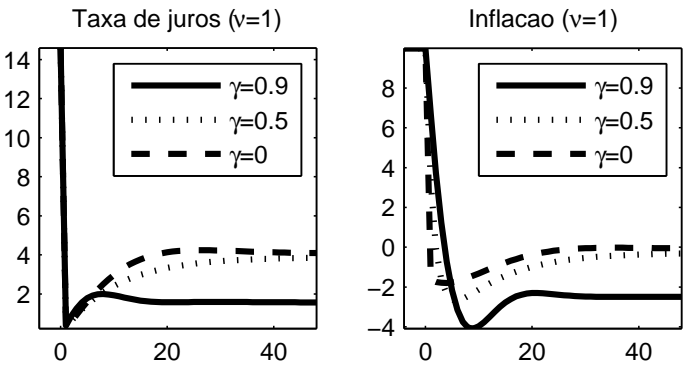

Taxa de juros real $(v=1)$
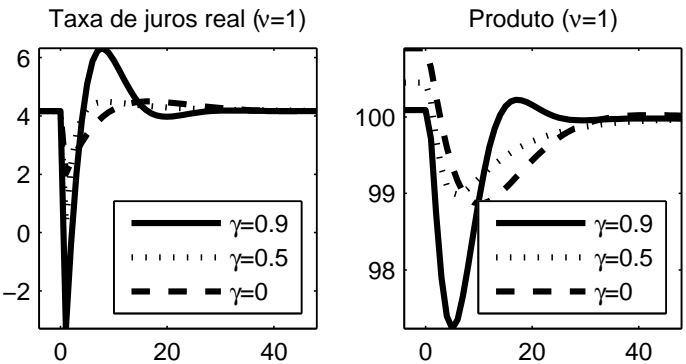

Taxa de juros $(v=1000)$

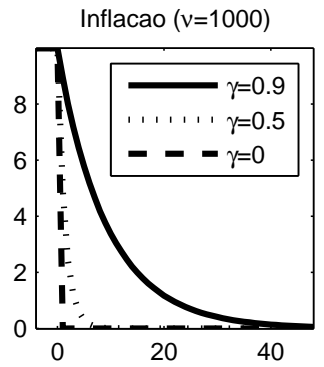

Taxa de juros real $(v=1000)$
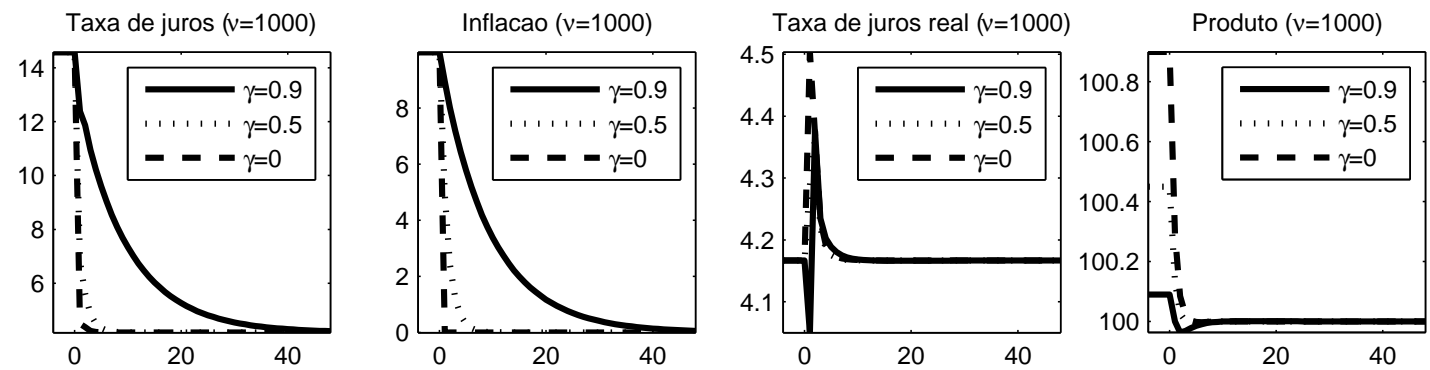

Obs.: Apresentam-se as trajetórias da taxa de juros nominal e real, inflação e produto sob diferentes magnitudes da velocidade da moeda $\nu=(\bar{Y} / \bar{M})$ - que assume os valores $0.36,1$ e 1000 nos gráficos da primeira, segunda e terceira linha, respectivamente - e sob diferentes graus de indexação: $\gamma=0.9$ (linhas sólidas), $\gamma=0.5$ (linhas pontilhadas) e $\gamma=0$ (linhas sólidas com marcadores).

Evidentemente, caso uma redução dessa magnitude na taxa de juros tivesse sido antecipada no período -1 , o produto teria subido fortemente, inviabilizando a redução da inflação.

Para fins de comparação, apresentam-se na Figura 5 as trajetórias obtidas nas mesmas condições da Figura 4, exceto pelo valor de $r^{*}$, suposto igual a zero. Notam-se duas diferenças significativas entre essas figuras: (i) a inflação converge, agora, para zero; (ii) na ausência de indexação, a inflação é estabilizada imediatamente e não há recessão, independentemente da magnitude das friç̧ões monetárias. O primeiro resultado é óbvio pela discussão anterior. Quanto ao segundo, a explicação baseia-se na ausência de dilema entre os objetivos de estabilização da inflação e dos juros.

Simulações adicionais, não reportadas por razões de espaço, mostram que a existência de formação de hábito não tem efeito relevante sobre o plano ótimo no caso em que as friç̧ões monetárias são pouco significativas, conforme já demonstrado anteriormente por Giannoni e Woodford (2004). No caso em que tais fricções são relevantes, porém, a introdução de formação de hábito pode gerar perdas adicionais de mais de $1 \%$ do produto, além de exacerbar o padrão cíclico do produto. Cabe ressaltar que 
Figura 5: Trajetórias sob a regra ótima de desinflação $\left(r^{*}=0\right)$
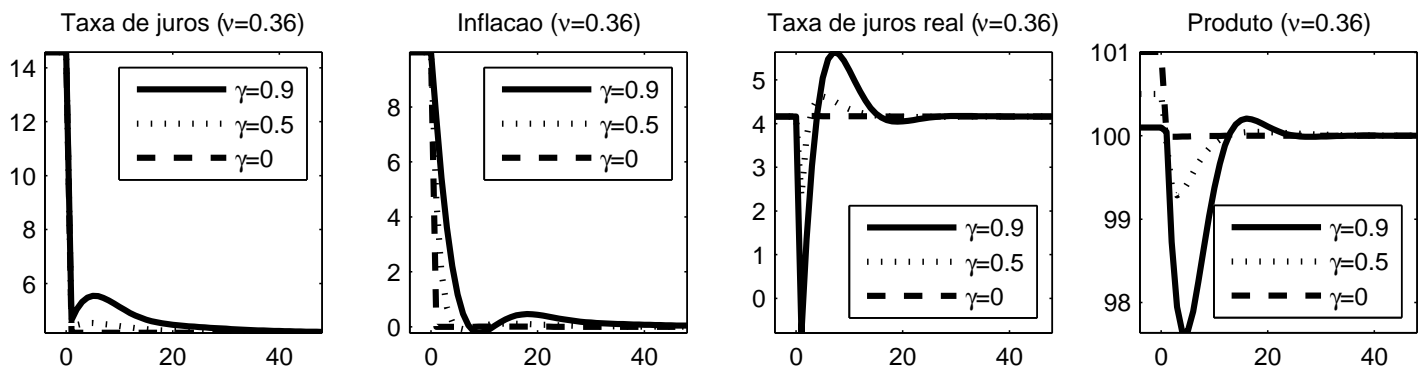

Taxa de juros $(v=1)$
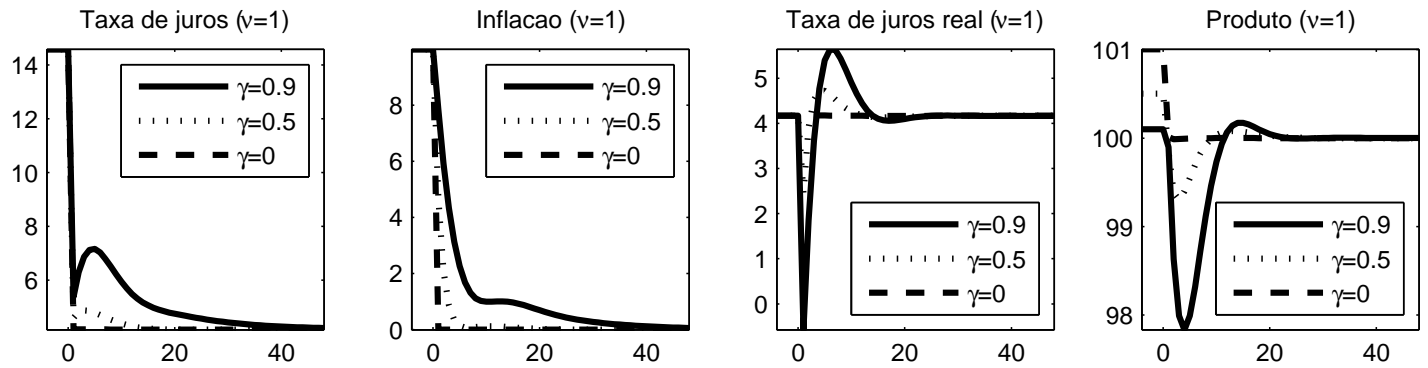

Taxa de juros $(v=1000)$
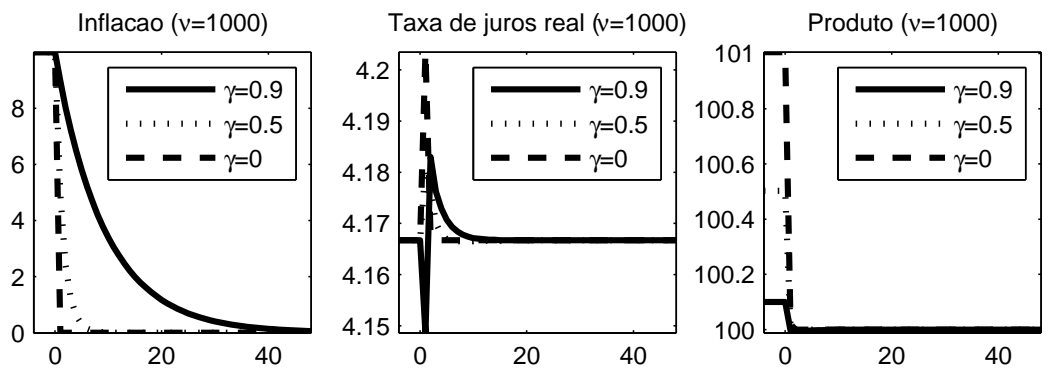

Obs.: Apresentam-se as trajetórias da taxa de juros nominal e real, inflação e produto sob diferentes magnitudes da velocidade da moeda $\nu=(\bar{Y} / \bar{M})$ - que assume os valores $0.36,1$ e 1000 nos gráficos da primeira, segunda e terceira linha, respectivamente - e sob diferentes graus de indexação: $\gamma=0.9$ (linhas sólidas), $\gamma=0.5$ (linhas pontilhadas) e $\gamma=0$ (linhas sólidas com marcadores).

os efeitos da formação de hábito revelam-se aqui consideravelmente mais significativos do que sob as regras arbitrárias da seção anterior.

Por fim, a Figura 6 compara a perda de bem-estar derivada sob a regra ótima com as perdas obtidas sob regras arbitrárias da classe (16a)-(16b), para valores de $a$ entre 0 e 0.99 e dois valores possíveis de $\psi$ (um valor 'alto' e um valor 'baixo'). O principal ponto a ser observado é que, em geral, o desempenho das regras arbitrárias pode aproximar-se arbitrariamente do desempenho da regra ótima pela escolha adequada do valor do parâmetro $a$. As exceções parecem ser os casos caracterizados por alto grau de indexação $e$ frições monetárias relevantes, em que as diferenças de desempenho revelam-se mais significativas (ainda que as diferenças possam parecer quantitativamente pequenas). A explicação para esse fenômeno está relacionada, conforme discutido anteriormente, ao fato de que, em economias com tais características, o dilema entre os vários objetivos de estabilização é particularmente significativo: de um lado, quanto maiores as friç̧ões monetárias, maiores os custos da manutenção de níveis elevados de inflação e juros e, portanto, maior o incentivo a desinflar rapidamente; de outro lado, quanto mais 
Figura 6: Perda de bem-estar: comparação entre a regra ótima e regras 'arbitrárias'
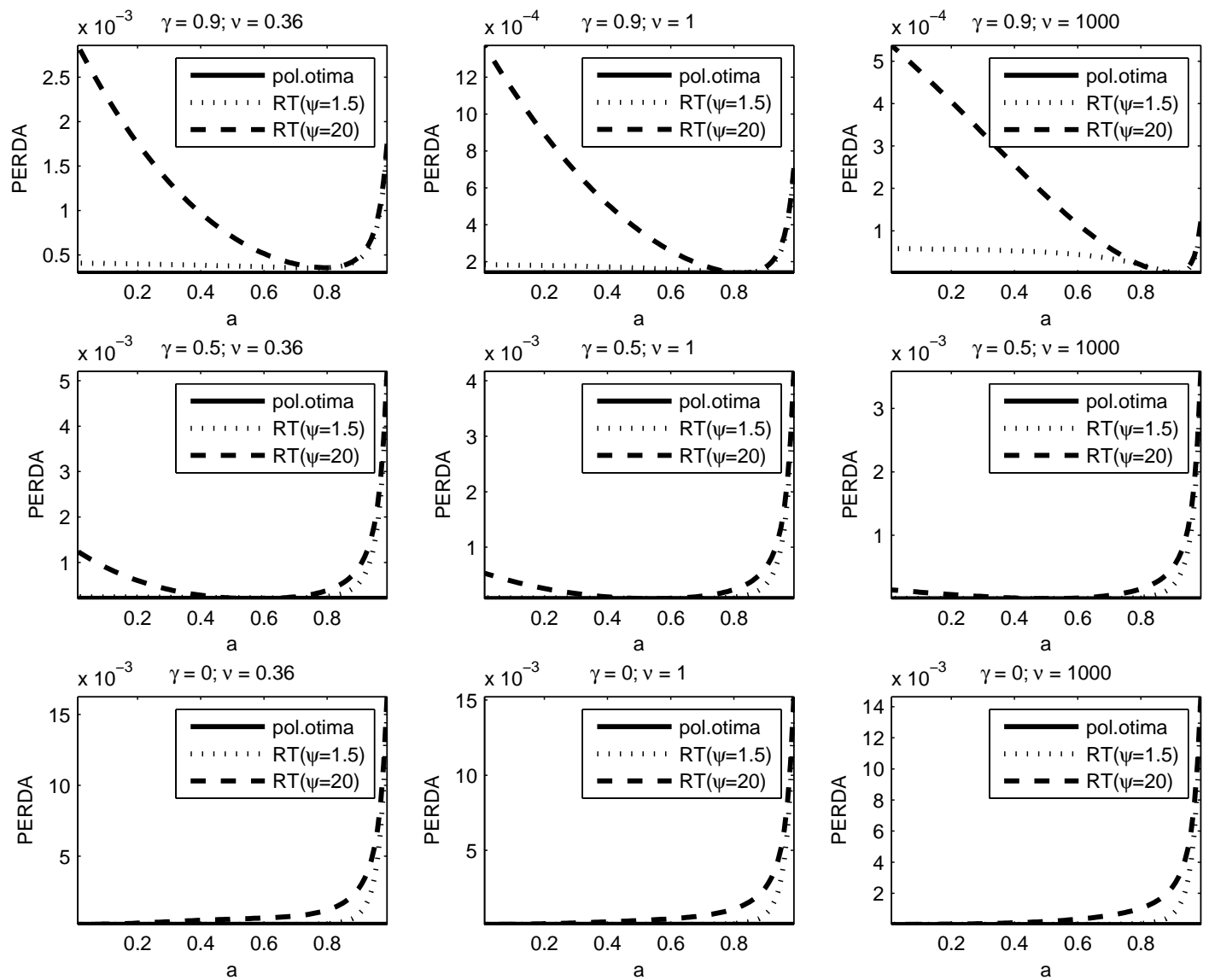

Obs.: Apresentam-se as perdas de bem-estar geradas pela regra ótima (linhas sólidas), pela regra arbitrária com $\psi=1.5$ (linhas pontilhadas) e pela regra arbitrária $\operatorname{com} \psi=20$ (linhas tracejadas), sob diferentes magnitudes da velocidade da moeda $\nu=(\bar{Y} / \bar{M})$ - que assume os valores $0.36,1$ e 1000 nos gráficos da primeira, segunda e terceira coluna, respectivamente -, e do grau de indexação da economia $\gamma$ - que assume os valores $0.9,0.5$ e 0 nos gráficos da primeira, segunda e terceira linha, respectivamente.

indexada a economia, maior a perda de bem-estar associada a mudanças bruscas na taxa de inflação e, consequentemente, melhor uma estratégia de desinflação gradual. De qualquer forma, é interessante notar que, sob diversas circunstâncias, uma trajetória de desinflação ‘quase tão boa como a ótima' poderia ser obtida pela implementação de uma regra muito simples - com todos os benefícios associados ao uso de regras simples, como transparência, facilidade de comunicação etc.

Os principais resultados desta seção podem ser assim resumidos:

i. A política ótima de desinflação nunca gera um boom e, em geral, envolve uma recessão inicial;

ii. O grau de indexação na economia afeta positivamente o tamanho da recessão inicial e negativamente a velocidade ótima de desinflação;

iii. À medida que a magnitude das fricções monetárias se eleva, aumentam o tamanho da recessão inicial requerida para desinflar a economia e a velocidade ótima de desinflação; 
iv. Quando as friç̧ões monetárias são irrelevantes e não há indexação, a desinflação ótima é imediata e apresenta custos mínimos em termos de perda de produto;

v. Sob friç̧ões monetárias relevantes, a introdução de formação de hábito gera perdas adicionais de produto significativas;

vi. A trajetória ótima das taxas de inflação e juros não é monotonicamente decrescente durante o processo de desinflação quando as fricções monetárias são relevantes;

vii. Exceto em economias com elevados níveis de indexação e fricções monetárias, o desempenho de regras arbitrárias de desinflação parece razoavelmente próximo do desempenho da regra ótima.

\section{CONCLUSÃO}

Este artigo analisou as propriedades das políticas ótimas de desinflação no contexto de um modelo monetário de equilíbrio geral 'novo-keynesiano' em economia fechada, buscando responder a duas perguntas em particular:

(i) A política ótima envolve uma desinflação 'rápida' ou 'gradual’?

(ii) A desinflação ótima requer uma recessão significativa?

De acordo com os resultados obtidos, a existência de diferentes graus de friç̧ões monetárias e de inércia no produto e na inflação permite justificar diferentes trajetórias ótimas de desinflação, algumas envolvendo queda rápida e relativamente indolor da inflação, outras associadas à lenta redução das taxas inflacionárias acompanhada de forte recessão. Mais especificamente, constatou-se que o tamanho da recessão inicial requerida para desinflar a economia aumenta com as fricções monetárias e com os graus de inércia no produto e na inflação, ao passo que a velocidade ótima de desinflação diminui com o grau de indexação na economia, mas aumenta com a magnitude das fricções monetárias.

$\mathrm{O}$ artigo tem três contribuições importantes. Primeiro, foram abordados aspectos relativamente inexplorados na literatura sobre desinflação, como o papel das fricções monetárias, e da interação destas com o grau de indexação da economia, na determinação da velocidade ótima de desinflação. Segundo, a análise de bem-estar esteve calcada nos mesmos microfundamentos subjacentes às equações estruturais do modelo, e não em critérios relativamente arbitrários usados na maioria dos outros estudos - como, por exemplo, a 'taxa de sacrifício'. Terceiro, a questão da desinflação foi analisada a partir de regras baseadas no controle da taxa de juros pelo Banco Central, e não simplesmente na determinação de trajetórias para a evolução do estoque de moeda na economia, como feito na maioria dos trabalhos sobre o tema. Tendo em vista que, de acordo com a prática corrente na condução da política monetária, o principal instrumento de política é a taxa de juros básica, essa opção metodológica revela-se importante na aplicação dos resultados para fins de recomendações de política.

Dentre as principais limitações do estudo, cabe citar que o modelo é construído para uma economia fechada e pressupõe credibilidade perfeita das autoridades monetárias - o que impede a consideração de aspectos importantes dos processos de desinflação em economias reais, associados, respectivamente, à evolução da taxa de câmbio e das expectativas dos agentes privados com relação à condução da política monetária. Pretende-se, no futuro, estender o modelo de modo a incorporar essas questões. 


\section{BIBLIOGRAFIA}

Amato, J. D. \& Laubach, T. (2003). Rule-of-thumb behaviour and monetary policy. European Economic Review, 47:791-831.

Ascari, G. (2004). Staggered prices and trend inflation: Some nuisances. Review of Economic Dynamics, $7(3): 642-667$.

Bakhshi, H., Burriel-Llombart, P., Khan, H., \& Rudolf, B. (2003). Endogenous price stickiness, trend inflation, and the New Keynesian Phillips curve. Bank of England Working Paper No. 191.

Ball, L. (1994a). Credible disinflation with staggered price-setting. The American Economic Review, 84(1):282-289.

Ball, L. (1994b). What determines the sacrifice ratio? In Mankiw, G. N., editor, Monetary Policy. University of Chicago Press, Chicago.

Ball, L. (1995). Disinflation and imperfect credibility. Journal of Monetary Economics, 35:5-24.

Blanchard, O. \& Kahn, C. (1980). The solution of linear difference models under rational expectations. Econometrica, 48:1305-1311.

Boivin, J. \& Giannoni, M. P. (2003). Has monetary policy become more effective? NBER Working Paper no. 9459.

Calvo, G. A. (1983). Staggered prices in a utility-maximizing framework. Journal of Monetary Economics, 12:383-398.

Calvo, G. A., Celasun, O., \& Kumhof, M. (2003). Inflation inertia and credible disinflation - The open economy case. NBER Working Paper No. 9557.

Christiano, L., Eichenbaum, M., \& Evans, C. (1999). Monetary policy shocks: What have we learned and to what end? In Taylor, J. B. \& Woodford, M., editors, HandBook of MacroEconomics, pages 65-148. Elsevier, North-Holland, Amsterdam.

Christiano, L., Eichenbaum, M., \& Evans, C. (2001). Nominal rigidities and the dynamic effects of a shock to monetary policy. National Bureau of Economic Research Working Paper 8403.

Danziger, L. (1988). Costs of price adjustment and the welfare economics of inflation and disinflation. American Economic Review, 78:633-646.

Erceg, C. J. \& Levin, A. T. (2003). Imperfect credibility and inflation persistence. Journal of Monetary Economics, 50:915-944.

Fuhrer, J. C. (2000). Habit formation in consumption and its implications for monetary-policy models. American Economic Review, 90(3):367-390.

Giannoni, M. \& Woodford, M. (2004). Optimal inflation-targeting rules. In Bernanke, B. S. \& Woodford, M., editors, The In Ation Targeting Debate, pages 93-162. University of Chicago Press, Chicago.

Huh, C. \& Lansing, K. (2000). Expectations, credibility, and disinflation in a small macroeconomic model. Journal of Economics and Business, 52(1-2):51-86.

Ireland, P. N. (1995). Optimal disinflationary paths. Journal of Economic Dynamics and Control, 19:14291448. 
Ireland, P. N. (1997). Stopping inflations, big and small. Journal of Money, Credit, and Banking, 29:759775.

Ireland, P. N. (2001). Money's role in the monetary business cycle. NBER Working Paper 8115.

King, R. G. \& Watson, M. W. (1998). The solution of singular linear difference systems under rational expectations. International Economic Review, 39(4):1015-26.

Rotemberg, J. \& Woodford, M. (1997). An optimization-based econometric framework for the evaluation of monetary policy. In Bernanke, B. S. \& Rotemberg, J. J., editors, NBER Macroeconomics Annual, pages 297-346. MIT Press, Cambridge.

Taylor, J. B. (1993). Discretion versus policy rules in practice. Carnegie-Rochester Conference Series on Public Policy, 39:195-214.

Walsh, C. (1998). Monetary Theory and Policy. MIT Press, Cambridge.

Woodford, M. (1999). Optimal monetary policy inertia. NBER Working Paper No. 7261.

Woodford, M. (2003). Interest and Prices: Foundations of a Theory of Monetary Policy. Princeton University Press, Princeton. 


\section{A. DERIVAÇÃO DO BLOCO DE DEMANDA}

O problema do consumidor é maximizar

$$
E_{0}\left\{\sum_{t=0}^{\infty} \beta^{t}\left[u\left(C_{t}^{i}, C_{t-1}^{i}, \tilde{M}_{t}^{i}\right)-v\left(y_{t}(i)\right)\right]\right\}
$$

sujeito à restrição orçamentária

$$
M_{t}+B_{t}+\int_{0}^{1} p_{t}(i) c_{t}(i) d i \leq P_{t} Y_{t}+T_{t}+M_{t-1} R_{t-1}^{m}+B_{t-1} R_{t-1}
$$

Inicialmente, note que $\int_{0}^{1} p_{t}(i) c_{t}(i) d i=P_{t} C_{t}$. De fato, sob a especificação de Dixit-Stiglitz, os índices de consumo e preço agregados são dados por

$$
\begin{aligned}
C_{t} & =\left[\int_{0}^{1} c_{t}(i)^{(\theta-1) / \theta} d i\right]^{\theta /(\theta-1)} \\
P_{t} & =\left[\int_{0}^{1} p_{t}(i)^{1-\theta} d i\right]^{1 /(1-\theta)}
\end{aligned}
$$

e a demanda de cada bem diferenciado deve satisfazer

$$
c_{t}(i)=C_{t}\left(\frac{p_{t}(i)}{P_{t}}\right)^{-\theta}
$$

Logo,

$$
\begin{aligned}
\int_{0}^{1} p_{t}(i) c_{t}(i) d i & =\int_{0}^{1} p_{t}(i) C_{t}\left(\frac{p_{t}(i)}{P_{t}}\right)^{-\theta} d i \\
& =C_{t} P_{t}^{\theta} \int_{0}^{1} p_{t}(i)^{1-\theta} d i \\
& =C_{t} P_{t}
\end{aligned}
$$

Usando o resultado acima, pode-se reescrever a restrição orçamentária do consumidor (A-2) em termos reais:

$$
\tilde{M}_{t}+\tilde{B}_{t}+C_{t} \leq Y_{t}+\tilde{T}_{t}+\tilde{M}_{t-1}\left(\frac{R_{t-1}^{m}}{\Pi_{t}}\right)+\tilde{B}_{t-1}\left(\frac{R_{t-1}}{\Pi_{t}}\right)
$$

onde $\tilde{M}_{t}$ e $\tilde{B}_{t}$ são os estoques reais de moeda e títulos, e $\Pi_{t}$ é a taxa de inflação (bruta) entre os períodos $t-1$ e $t$. O problema do consumidor é, assim, escolher trajetórias $\left\{C_{t}\right\},\left\{\tilde{M}_{t}\right\}$ e $\left\{\tilde{B}_{t}\right\}$ para $t \geq 0$ de modo a maximizar (A-1) sujeito a (A-3). As condições de primeira ordem são:

$$
\begin{aligned}
u_{1}\left(C_{t}, C_{t-1}, \tilde{M}_{t}\right)+\beta E_{t}\left[u_{2}\left(C_{t+1}, C_{t}, \tilde{M}_{t+1}\right)\right]-\Lambda_{t} & =0 \\
u_{3}\left(C_{t}, C_{t-1}, \tilde{M}_{t}\right)-\Lambda_{t}+\beta E_{t}\left[\frac{R_{t}^{m}}{\Pi_{t+1}} \Lambda_{t+1}\right] & =0 \\
\Lambda_{t}-\beta E_{t}\left[\frac{R_{t}}{\Pi_{t+1}} \Lambda_{t+1}\right] & =0
\end{aligned}
$$


onde $\Lambda_{t}$ é o multiplicador de Lagrange associado à restrição orçamentária no período t e $u_{k}($.$) é a$ derivada parcial de $u($.$) em relação ao k-ésimo argumento. Além dessas condições, supõe-se que seja$ satisfeita a condição de transversalidade relevante. Eliminando $\Lambda_{t}$ desse sistema, obtém-se

$$
\begin{aligned}
E_{t}\left[\frac{u_{1}\left(C_{t}, C_{t-1}, \tilde{M}_{t}\right)+\beta u_{2}\left(C_{t+1}, C_{t}, \tilde{M}_{t+1}\right)}{u_{1}\left(C_{t+1}, C_{t}, \tilde{M}_{t+1}\right)+\beta u_{2}\left(C_{t+2}, C_{t+1}, \tilde{M}_{t+2}\right)}\right] & =\beta E_{t} \frac{R_{t}}{\Pi_{t+1}} \\
\frac{u_{3}\left(C_{t}, C_{t-1}, \tilde{M}_{t}\right)}{u_{1}\left(C_{t}, C_{t-1}, \tilde{M}_{t}\right)+\beta E_{t} u_{2}\left(C_{t+1}, C_{t}, \tilde{M}_{t+1}\right)} & =\frac{R_{t}-R_{t}^{m}}{R_{t}}
\end{aligned}
$$

Impondo a condição de equilíbrio no mercado de bens, que requer $C_{t}=Y_{t}$ para todo t, e loglinearizando (A-7) e (A-8) em torno do equilíbrio com inflação zero $(\bar{\Pi}=1)$, chega-se ao bloco de demanda do modelo:

$$
\begin{aligned}
y_{t}= & \delta_{1} y_{t-1}+\delta_{2} E_{t} y_{t+1}+\delta_{3} E_{t} y_{t+2}+\delta_{4}\left(r_{t}-E_{t} \pi_{t+1}\right) \\
& +\delta_{5} m_{t}+\delta_{6} E_{t} m_{t+1}+\delta_{7} E_{t} m_{t+2} \\
m_{t}= & \eta_{1} y_{t}+\eta_{2} y_{t-1}+\eta_{3} E_{t} y_{t+1}+\eta_{4} r_{t}+\eta_{5} E_{t} m_{t+1}
\end{aligned}
$$

onde

$$
\begin{array}{rlrl}
\delta_{1} & =\frac{-\rho \epsilon_{12}}{A} & \eta_{1}=\frac{\rho \epsilon_{11}-\epsilon_{31}+(1-\rho) \epsilon_{22}}{B} \\
\delta_{2}=\frac{\rho \epsilon_{11}-(1-\rho)\left(\epsilon_{21}-\epsilon_{22}\right)}{A} & \eta_{2}=\frac{\rho \epsilon_{12}-\epsilon_{32}}{B} \\
\delta_{3}=\frac{(1-\rho) \epsilon_{21}}{A} & \eta_{3}=\frac{(1-\rho) \epsilon_{21}}{B} \\
\delta_{4}=\frac{1}{A} & \eta_{4}=\left(\frac{\bar{R}^{m}}{\bar{R}-\bar{R}^{m}}\right) \frac{1}{B} \\
\delta_{5}=\frac{-\rho \epsilon_{13}}{A} & \eta_{5}=\frac{(1-\rho) \epsilon_{23}}{B} \\
\delta_{6}=\frac{\rho \epsilon_{13}-(1-\rho) \epsilon_{23}}{A} & & \\
\delta_{7}=\frac{(1-\rho) \epsilon_{23}}{A} & &
\end{array}
$$

e

$$
\begin{aligned}
& A=(1-\rho) \epsilon_{22}+\rho\left(\epsilon_{11}-\epsilon_{12}\right) \\
& B=\epsilon_{33}-\rho \epsilon_{13} \\
& \rho=\frac{u_{1}(\bar{C}, \bar{C}, \bar{M})}{u_{1}(\bar{C}, C, M)+\beta u_{2}(C, C, M)}
\end{aligned}
$$

e $\epsilon_{i j}$ é a elasticidade de $u_{i}$ em relação ao j-ésimo argumento de $u($.$) , avaliada no equilíbrio de estado$ estacionário. Para fins da derivação do critério de bem-estar mais adiante, é interessante reescrever a equação de demanda de moeda em função de elasticidades-renda e juros:

$$
m_{t}=\eta_{y}(L) y_{t}-\eta_{r}(L) r_{t}
$$

onde

$$
\begin{aligned}
\eta_{y}(L) & =\frac{\eta_{1}+\eta_{2} L+\eta_{3} L^{-1}}{1-\eta_{5} L^{-1}} \\
\eta_{r}(L) & =\frac{-\eta_{4}}{1-\eta_{5} L^{-1}}
\end{aligned}
$$

sendo $L$ o operador de defasagem (e $L^{-1}$, portanto, o operador de avanço). 


\section{B. DERIVAÇÃO DA EQUAÇÃO DE OFERTA}

Os ofertantes sorteados no período t devem escolher $P_{t}^{*}$ de modo a maximizar

$$
\begin{array}{r}
E_{t} \sum_{j=0}^{\infty}(\alpha \beta)^{j}\left\{\Lambda_{t+j}\left[\frac{P_{t}^{*}}{P_{t+j}}\left(\frac{P_{t+j-1}}{P_{t-1}}\right)^{\gamma}\right]^{1-\theta} Y_{t+j}\right. \\
\left.-v\left[\left[\frac{P_{t}^{*}}{P_{t+j}}\left(\frac{P_{t+j-1}}{P_{t-1}}\right)^{\gamma}\right]^{-\theta} Y_{t+j}\right]\right\}
\end{array}
$$

A condição de primeira ordem desse problema conduz, após algumas manipulações algébricas, à seguinte expressão para o preço relativo ótimo $\tilde{P}_{t}^{*}=P_{t}^{*} / P_{t}$ :

$$
\tilde{P}_{t}^{*}=\frac{\theta}{\theta-1} \frac{E_{t} \sum_{j=0}^{\infty}(\alpha \beta)^{j} v_{1} Y_{t+j}\left(\prod_{i=1}^{j} \Pi_{t+i-1}\right)^{-\gamma \theta}\left(\prod_{i=1}^{j} \Pi_{t+i}\right)^{\theta}}{E_{t} \sum_{j=0}^{\infty}(\alpha \beta)^{j} \Lambda_{t+j} Y_{t+j}\left(\prod_{i=1}^{j} \Pi_{t+i-1}\right)^{\gamma-\gamma \theta}\left(\prod_{i=1}^{j} \Pi_{t+i}\right)^{\theta-1}}
$$

A dinâmica do índice de preço agregado $P_{t}$ pode então ser derivada. Dado que todos os ofertantes sorteados na data $t$ escolhem o mesmo preço ótimo $P_{t}^{*}$, pois se deparam com o mesmo fluxo de lucros instantâneos esperados, e a probabilidade $\alpha$ de um ofertante não ser sorteado é constante no tempo e independente de seu histórico de ajuste de preços, o preço agregado na data $t$ será dado por

$$
P_{t}=\left\{(1-\alpha) P_{t}^{*(1-\theta)}+\alpha\left[P_{t-1}\left(\frac{P_{t-1}}{P_{t-2}}\right)^{\gamma}\right]^{(1-\theta)}\right\}^{1 /(1-\theta)}
$$

A log-linearização e manipulação das equações (B-13) e (B-14) conduzirá à equação de oferta agregada do modelo. Primeiro, log-linearizando a equação (B-13), obtém-se:

$$
\tilde{p}_{t}^{*}=\left(\frac{1-\alpha \beta}{1+\theta \omega}\right) E_{t} \sum_{j=0}^{\infty}(\alpha \beta)^{j}\left[(1+\theta \omega) \sum_{i=1}^{j}\left(\pi_{t+i}-\gamma \pi_{t+i-1}\right)+\omega y_{t+j}-\lambda_{t+j}\right]
$$

onde $\tilde{p}_{t}^{*}$ e $\lambda_{t}$ são os desvios percentuais de $\tilde{P}_{t}^{*}$ e $\Lambda_{t}$ em relação a seus valores no equilíbrio estacionário, e $\omega$ é a elasticidade de $v_{1}$ em relação ao produto. A partir de (B-15), pode-se calcular a quase-diferença

$$
\tilde{p}_{t}^{*}-\alpha \beta E_{t} \tilde{p}_{t+1}^{*}=\left(\frac{1-\alpha \beta}{1+\theta \omega}\right)\left(\omega y_{t}-\lambda_{t}\right)+\alpha \beta\left(E_{t} \pi_{t+1}-\gamma \pi_{t}\right)
$$

que, combinada com a log-linearização de (B-14),

$$
\tilde{p}_{t}^{*}=\left(\frac{\alpha}{1-\alpha}\right)\left(\pi_{t}-\gamma \pi_{t-1}\right),
$$

e com a expressão para $\lambda_{t}$ obtida a partir da log-linearização de (A-4),

$$
\begin{aligned}
\lambda_{t}= & {\left[\rho \epsilon_{11}+(1-\rho) \epsilon_{22}\right] y_{t}+\rho \epsilon_{12} y_{t-1}+(1-\rho) \epsilon_{21} E_{t} y_{t+1} } \\
& +\rho \epsilon_{13} m_{t}+(1-\rho) \epsilon_{23} E_{t} m_{t+1}
\end{aligned}
$$

leva à curva de Phillips 


$$
\begin{aligned}
\pi_{t}-\gamma \pi_{t-1}= & \mu_{1} y_{t}+\mu_{2} y_{t-1}+\mu_{3} E_{t} y_{t+1}+\mu_{4} m_{t}+\mu_{5} E_{t} m_{t+1} \\
& +\beta\left(E_{t} \pi_{t+1}-\gamma \pi_{t}\right)
\end{aligned}
$$

onde

$$
\begin{aligned}
& \mu_{1}=\tilde{\kappa}\left[\omega-\rho \epsilon_{11}-(1-\rho) \epsilon_{22}\right] \\
& \mu_{2}=-\tilde{\kappa} \rho \epsilon_{12} \\
& \mu_{3}=-\tilde{\kappa}(1-\rho) \epsilon_{21} \\
& \mu_{4}=-\tilde{\kappa} \rho \epsilon_{13} \\
& \mu_{5}=-\tilde{\kappa}(1-\rho) \epsilon_{23}
\end{aligned}
$$

e

$$
\tilde{\kappa}=\frac{(1-\alpha)(1-\alpha \beta)}{\alpha(1+\theta \omega)} .
$$




\section{DERIVAÇÃO DO CRITÉRIO DE BEM-ESTAR}

O critério de bem-estar relevante é derivado a partir de uma aproximação de Taylor de segunda ordem da utilidade esperada do agente representativo:

$$
W=E\left\{\sum_{t=0}^{\infty} \beta^{t} U_{t}\right\}
$$

onde

$$
U_{t}=u\left(Y_{t}, Y_{t-1}, \tilde{M}_{t}\right)-\int_{0}^{1} v\left(y_{t}(i)\right) d i
$$

A inclusão da integral em $i$ no objetivo acima permite tomar a média da desutilidade do trabalho entre os agentes em cada ponto do tempo e, assim, desconsiderar os efeitos de diferentes históricos de mudanças de preços sobre a oferta de cada agente.

A aproximação de Taylor de segunda ordem do objetivo (C-20) toma por base o mesmo equilíbrio de estado estacionário em torno do qual as equações estruturais foram log-linearizadas - isto é, o equilíbrio com inflação zero. Supõe-se, ademais, que: (i) as políticas consideradas gerem resultados 'suficientemente próximos' desse estado estacionário; (ii) a produção em estado estacionário receba um montante de subsídios suficiente para que o equilíbrio se caracterize por um nível eficiente de produto, apesar da existência de competição imperfeita no mercado de bens; (iii) no estado estacionário, a economia se encontre suficientemente próxima do nível (finito) de saciedade em termos de saldos monetários reais. Essas hipóteses são necessárias para garantir a validade da aproximação de segunda ordem da utilidade esperada (C-20) quando esta é avaliada a partir da solução do sistema de relações lineares (6), (7) e (11) - acrescidas de uma regra de política monetária - que são aproximações das equações estruturais exatas.

A hipótese de 'saciedade aproximada' em relação a saldos monetários reais equivale a considerar uma série de economias que se aproximem arbitrariamente do limite caracterizado pelas condições

$$
\begin{aligned}
u_{3}(\bar{Y}, \bar{Y}, \bar{M}) & \longrightarrow 0 \\
u_{13}(\bar{Y}, \bar{Y}, \bar{M}) & \longrightarrow 0 \\
u_{23}(\bar{Y}, \bar{Y}, \bar{M}) & \longrightarrow 0 \\
u_{33}(\bar{Y}, \bar{Y}, \bar{M}) & \longrightarrow c t e<0
\end{aligned}
$$

que, por sua vez, implicam

$$
\begin{array}{ll}
\epsilon_{13} \longrightarrow 0 & \epsilon_{33} \longrightarrow-\infty \\
\epsilon_{23} \longrightarrow 0 & \eta_{y} \longrightarrow 0 \\
\epsilon_{31} \longrightarrow c t e>0 & \eta_{r} \longrightarrow c t e>0 \\
\epsilon_{32} \longrightarrow c t e &
\end{array}
$$

Alternativamente, a saciedade em saldos monetários pode ser retratada pela 'regra de Friedman'

$$
\bar{R} \longrightarrow \bar{R}^{m}
$$

Com o objetivo de simplificar a exposição, omite-se a derivação detalhada da expansão de Taylor de segunda ordem da utilidade esperada; a seguir, são apresentados apenas os principais passos desse procedimento. O ponto de partida é a aproximação do primeiro termo de $U_{t}$, que leva a 


$$
\begin{aligned}
u\left(Y_{t}, Y_{t-1}, \tilde{M}_{t}\right)= & \bar{u}+\bar{u}_{1} \bar{Y}\left\{y_{t}+\left(\frac{1+\epsilon_{11}}{2}\right) y_{t}^{2}+\frac{\epsilon_{12}}{\epsilon_{21}} y_{t-1}\right. \\
+ & \frac{\epsilon_{12}}{\epsilon_{21}}\left(\frac{1+\epsilon_{22}}{2}\right) y_{t-1}^{2}+\epsilon_{12} y_{t} y_{t-1}+\frac{\epsilon_{13}}{\epsilon_{31}} m_{t} \\
& \left.+\frac{\epsilon_{13}}{\epsilon_{31}}\left(\frac{1+\epsilon_{33}}{2}\right) m_{t}^{2}+\epsilon_{13} y_{t} m_{t}+\frac{\epsilon_{13} \epsilon_{32}}{\epsilon_{31}} y_{t-1} m_{t}\right\} \\
& +O\left(\|\hat{y}, \hat{m}\|^{3}\right)
\end{aligned}
$$

onde $\bar{u}=u(\bar{Y}, \bar{Y}, \bar{M})$ e, como definido anteriormente, todas as elasticidades $\epsilon_{i j}$ estão avaliadas no equilíbrio de estado estacionário. O resíduo dessa aproximação é de ordem $O\left(\|\hat{y}, \hat{m}\|^{3}\right)$, onde $\|\hat{y}, \hat{m}\|$ designa o limite para as flutuações do produto e dos saldos reais em relação a seus valores em estado estacionário. Substituindo, em (C-23),os termos em $m_{t}$ pela expressão dada em (A-11) e usando as condições (C-21a)-(C-21d) associadas à hipótese de saciedade de saldos monetários, obtém-se

$$
\begin{aligned}
u\left(Y_{t}, Y_{t-1}, \tilde{M}_{t}\right)= & \bar{u}+\bar{u}_{1} \bar{Y}\left\{y_{t}+\left(\frac{1+\epsilon_{11}}{2}\right) y_{t}^{2}\right. \\
& +\frac{\epsilon_{12}}{\epsilon_{21}} y_{t-1}+\frac{\epsilon_{12}}{\epsilon_{21}}\left(\frac{1+\epsilon_{22}}{2}\right) y_{t-1}^{2}+\epsilon_{12} y_{t} y_{t-1} \\
& \left.-\frac{\epsilon_{13}}{\epsilon_{31}} \eta_{r} r_{t}+\frac{\epsilon_{13} \epsilon_{33}}{\epsilon_{31}}\left(\frac{\eta_{r}^{2}}{2}\right) r_{t}^{2}\right\}+O\left(\|\hat{y}, \hat{m}, \bar{\Delta}\|^{3}\right)
\end{aligned}
$$

onde o diferencial de juros $\bar{\Delta}=\left(\bar{R}-\bar{R}^{m}\right) / \bar{R}$ aparece como parâmetro de expansão adicional.

A aproximação do segundo termo de $U_{t}$ é dada por:

$$
\begin{aligned}
\int_{0}^{1} v\left(y_{t}(i)\right) d i= & \bar{v}+\frac{\bar{u}_{1} \bar{Y}}{\rho}\left\{y_{t}+\left(\frac{1+\omega}{2}\right) y_{t}^{2}+\frac{\theta(1+\theta \omega)}{2} \operatorname{var}_{i} \log p_{t}^{i}\right\} \\
& +O\left(\|\hat{y}\|^{3}\right)
\end{aligned}
$$

onde $\bar{v}=v(\bar{Y})$ e $v a r_{i} \log p_{t}^{i}$ é a variância, em corte transversal, dos preços dos produtos diferenciados na data $t$. Essa expressão indica ser ótimo reduzir o grau de dispersão de preços na economia (medido pelo termo $\operatorname{var}_{i} \log p_{t}^{i}$ ); sob o mecanismo de ajustamento de preço de Calvo, pode-se mostrar que a dispersão de preços depende das variações no índice de preço agregado e que, portanto, a política ótima deve envolver a estabilização da taxa de inflação na economia. De fato, usando (C-24) e (C-25) é possível mostrar que a aproximação do critério de bem-estar (3) é dada por:

$$
\begin{aligned}
\sum_{t=0}^{\infty} \beta^{t} U_{t}= & -\Omega \sum_{t=0}^{\infty} \beta^{t}\left[\left(\pi_{t}-\gamma \pi_{t-1}\right)^{2}+\lambda_{y}\left(y_{t}-\zeta y_{t-1}\right)^{2}+\lambda_{r}\left(r_{t}-r^{*}\right)^{2}\right] \\
& +t i p+O\left(\|\hat{y}, \bar{\Delta}\|^{3}\right)
\end{aligned}
$$


onde

$$
\begin{aligned}
\lambda_{y} & =\frac{\tilde{\kappa}}{\theta}\left(\frac{\rho \epsilon_{12}}{\zeta}\right)>0 \\
\lambda_{r} & =\frac{\tilde{\kappa}}{\theta} \frac{\bar{M}}{\bar{Y}} \eta_{r}>0 \\
r^{*} & =-\left(\frac{1}{\epsilon_{33} \eta_{4}}\right)=\frac{\bar{R}^{m}-\bar{R}}{\bar{R}^{m}}<0 \\
\Omega & =\frac{\bar{u}_{1} \bar{Y}}{2 \rho} \frac{\theta}{\tilde{\kappa}}>0
\end{aligned}
$$

e $\zeta$ é um parâmetro entre 0 e 1, derivado de modo semelhante ao adotado em Woodford (2003, cap. 5 e Apêndice E): primeiro, usando (C-24) e (C-24), escreve-se $\sum_{t=0}^{\infty} \beta^{t} U_{t}$ em função de $\sum_{t=0}^{\infty} \beta^{t}$ $\left(a_{1} y_{t}^{2}+a_{2} y_{t} y_{t-1}\right)$, onde $a_{1}$ e $a_{2}$ são funções dos parâmetros estruturais; segundo, igualando os coeficientes de $y_{t}^{2}$ e $y_{t} y_{t-1}$ nos dois lados da expressão abaixo,

$$
\begin{aligned}
\sum_{t=0}^{\infty} \beta^{t}\left(a_{1} y_{t}^{2}+a_{2} y_{t} y_{t-1}\right) & =\sum_{t=0}^{\infty} \beta^{t} a_{3}\left(y_{t}-a_{4} y_{t-1}\right)^{2} \\
& =\sum_{t=0}^{\infty} \beta^{t} a_{3}\left[\left(1+a_{4}^{2} \beta\right) y_{t}^{2}-2 a_{4} y_{t} y_{t-1}\right]
\end{aligned}
$$

obtém-se uma equação quadrática para $a_{4}$; o termo $\zeta$ corresponde à menor raiz dessa equação. 0 termo tip refere-se a 'termos independentes de política', isto é, termos cuja evolução não pode ser afetada pela política econômica - e que, portanto, podem ser desconsiderados no processo de avaliação de políticas alternativas.

Evidentemente, maximizar (C-26) é equivalente a minimizar

$$
\sum_{t=0}^{\infty} \beta^{t}\left[\left(\pi_{t}-\gamma \pi_{t-1}\right)^{2}+\lambda_{y}\left(y_{t}-\zeta y_{t-1}\right)^{2}+\lambda_{r}\left(r_{t}-r^{*}\right)^{2}\right]
$$

que é a 'função perda' usada no texto. 


\section{DERIVAÇÃO DA POLÍTICA ÓTIMA DE DESINFLAÇÃO}

A política ótima de desinflação é derivada a partir da minimização da função de perda (13), sujeita às restrições impostas pela equações estruturais (6) e (11). O Lagrangeano correspondente a esse problema é

$$
\begin{aligned}
\Delta= & \sum_{t=0}^{\infty} \beta^{t}\left\{\tilde{\pi}_{t}^{2}+\lambda_{y} \tilde{y}_{t}^{2}+\lambda_{r}\left(r_{t}-r^{*}\right)^{2}\right. \\
& +2 \phi_{1, t}\left[\tilde{\pi}_{t}-\mu_{1} y_{t}-\mu_{2} y_{t-1}-\mu_{3} y_{t+1}-\beta \tilde{\pi}_{t+1}\right] \\
& \left.+2 \phi_{2, t}\left[y_{t}-\delta_{1} y_{t-1}-\delta_{2} y_{t+1}-\delta_{3} y_{t+2}-\delta_{4}\left(r_{t}-\pi_{t+1}\right)\right]\right\}
\end{aligned}
$$

onde $\tilde{\pi}_{t}=\pi_{t}-\gamma \pi_{t-1}$ e $\tilde{y}_{t}=y_{t}-\zeta y_{t-1}$.

As condições de primeira ordem são:

$$
\begin{aligned}
0= & \left(1-\beta \gamma L^{-1}\right)\left(\tilde{\pi}_{t}+\phi_{1, t}-\phi_{1, t-1}\right)+\frac{\delta_{4}}{\beta} \phi_{2, t-1} \\
0= & \lambda_{y}\left(1-\beta \zeta L^{-1}\right) \tilde{y}_{t}-\left[\mu_{1}+\beta \mu_{2} L^{-1}+\frac{\mu_{3}}{\beta} L\right] \phi_{1, t} \\
& -\left[1+\beta \delta_{1} L^{-1}+\frac{\delta_{2}}{\beta} L+\frac{\delta_{3}}{\beta^{2}} L^{2}\right] \phi_{2, t} \\
0= & \phi_{2, t}-\frac{\lambda_{r}}{\delta_{4}}\left(r_{t}-r^{*}\right)
\end{aligned}
$$

que valem para $t \geq 0$, sujeito às condições iniciais

$$
\phi_{1,-1}=\phi_{2,-1}=\phi_{2,-2}=0
$$

Consideram-se apenas soluções limitadas para essas equações, de modo que não é necessário levar em consideração as condições de transversalidade para esse problema. O plano ótimo é, assim, dado por trajetórias para as variáveis $\pi_{t}, y_{t}, r_{t}, \phi_{1 t}$ e $\phi_{2 t}$ para todo $t \geq 0$, tais que sejam satisfeitas as equações (6), (11), (D-29a), (D-29b) e (D-29c) e as condições iniciais (D-30).

A imposição dessas condições iniciais deve-se ao fato de que, sendo a política implementada a partir da data 0 , a relação IS e a curva de Phillips nos períodos anteriores não impõem qualquer restrição ao problema de otimização. Evidentemente, isso implica, em geral, a inconsistência temporal da política considerada. Conforme nota Woodford (1999) em um contexto semelhante, a solução do problema de otimização (D-28) em qualquer data $t>0$ envolveria escolher trajetórias que satisfizessem (6), (11), (D-29a), (D-29b) e (D-29c) e condições iniciais $\phi_{1, t-1}=\phi_{2, t-1}=\phi_{2, t-2}=0$. Mas é fácil ver que essas condições iniciais não são satisfeitas pelo plano ótimo escolhido na data 0 . Fica claro, assim, que a hipótese de que as autoridades monetárias são capazes de comprometer-se a seguir o plano ótimo nas datas futuras é crucial para os resultados obtidos.

Após algumas manipulações, as condições de primeira ordem acima dão origem à 'regra instrumental implícita'

$$
\tilde{\pi}_{t}+\Phi_{y}(L) \tilde{y}_{t}=\Phi_{r}(L)\left(r_{t}-r^{*}\right)
$$

onde

$$
\begin{aligned}
& \Phi_{y}(L)=\lambda_{y}\left[\frac{\left(1-\beta \zeta L^{-1}\right)(1-L)}{\mu_{1}+\beta \mu_{2} L^{-1}+\beta^{-1} \mu_{3}}\right] \\
& \Phi_{r}(L)=\lambda_{r}\left[\frac{\left(1+\beta \delta_{1} L^{-1}+\beta^{-1} \delta_{2} L+\beta^{-2} \delta_{3} L^{2}\right)(1-L)}{\delta_{4}\left(\mu_{1}+\beta \mu_{2} L^{-1}+\beta^{-1} \mu_{3}\right)}-\frac{\beta^{-1} L}{1-\beta \gamma L^{-1}}\right]
\end{aligned}
$$

que resume o comportamento a ser adotado pelas autoridades monetárias em todos os períodos $t \geq 2$; para os períodos $t=0,1$ a regra deve ser modificada em função das condições iniciais (D-30). 